\title{
RESIDUES OF THE BOTT CLASS AND AN APPLICATION TO THE FUTAKI INVARIANT*
}

\author{
TARO ASUKE ${ }^{\dagger}$
}

\begin{abstract}
The Bott class for transversely holomorphic foliations and the Futaki invariant for complex manifolds are discussed. The main tools are a generalization of Heitsch's residue and the integration of Cech-de Rham cochains introduced by Lehmann. It is shown that the Bott class admits a natural decomposition as a differential character in the sense of Cheeger and Simons. The decomposition implies that the real and the complex parts of the Futaki invariant have a certain difference from the viewpoint of dynamical systems.
\end{abstract}

Introduction. In the theory of characteristic classes, so-called Chern-Simons classes are very important. In this paper, the Futaki invariant and more generally, the Bott class of transversely holomorphic foliations are studied. The Bott class is the most fundamental characteristic class for transversely holomorphic foliations. Here a foliation is said to be transversely holomorphic if its holonomy is written in terms of biholomorphic diffeomorphisms. The Bott class is a Chern-Simons class or CheegerSimons class for the complex normal bundle of the foliation. Under an additional assumption on the complex normal bundle, the definition of the Bott class becomes completely parallel to that of the Godbillon-Vey class. In this sense, the Bott class is a counterpart of the Godbillon-Vey class in the complex category. However, its properties are not yet well-understood. One of difficulties is that the Bott class is an element of $\boldsymbol{C} / \boldsymbol{Z}$-coefficient cohomology class. Indeed, the imaginary part can be written by a closed form and is easier to study, while the study of the real part often needs some additional techniques or notions, e.g. $\eta$-invariants. In this paper, first we introduce a modified version of Čech-de Rham complex and show that a certain representative of the Bott class can be treated as a differential form (Definition 2.13 and Theorem 2.19). This is based on the Chern-Weil theory on the Cech-de Rham complex found in [23]. There have been already some approaches to consider the de Rham cohomology with coefficients other than $\boldsymbol{C}$ or $\boldsymbol{R}$ [11], [21], where simplicial complexes are used. Under our formulation, the integration of Cech-de Rham cochains introduced by Lehmann [19] plays an essential role and it allows the construction to be elementary.

One of the main results is a generalization of Heitsch's residue of the Bott class (Theorem 3.4). Combined with a structure theorem of complex codimension-one foliations due to Ghys-Gomez-Mont-Saludes, this residue gives a generalization of Duminy's theorem in a weak form (Corollary 5.4). As an application of the residue to higher codimensional foliations, the Futaki invariant is discussed. It is a group character defined on the groups of biholomorphic automorphisms of a complex manifold and is valued in $\boldsymbol{C} \boldsymbol{Z}$. Given an automorphism $\sigma$ of a complex manifold $M$, one can naturally construct a foliated manifold $M_{\sigma}$ called the suspension, and the Futaki invariant is essentially the Bott class of this foliation. Calculations of the Futaki invariant often involve the $\eta$-invariant, which need some additional structures. The calculations

* Received March 10, 2003; accepted for publication May 30, 2003.

† Department of Mathematics (Yoshida-Minami branch), Kyoto University, Kyoto 606-8501, Japan (asuke@math.kyoto-u.ac.jp). Supported by Ministry of Education, Culture, Sports, Science and Technology, Grant No. 13740042, 15740036. 
presented in this paper do not need such structures and they are elementary though its amount is larger.

There is another type of residues based on a property of transversely Hermitian foliations, which is already introduced for the imaginary part of the Bott class in [2]. It is defined after showing a natural decomposition of the Bott class as a differential character in the sense of Cheeger-Simons [8] (Proposition 4.10, Definition 5.1). These two residues are in a similar relation as in the case of the imaginary part of the Bott class (Theorem 5.2). The decomposition is significant when the Futaki invariant is discussed. It gives in fact the decomposition of the Futaki invariant into the real and the imaginary parts, and it will be shown that the dependence of the real part of the Futaki invariant on the dynamics of the given automorphism is weaker than that of the imaginary part (Corollary 6.4).

This paper is organized as follows. In the first section, a modified Čech-de Rham complex is introduced and some facts concerning differential characters are recalled. The second section concerns the Bott class of transversely holomorphic foliations. A cocycle representing the Bott class is defined and its several properties are also studied. In the third section, a generalization of Heitsch's residue is defined. Certain properties of the Bott class as a differential character is discussed in Section 4. Section 5 concerns the complex codimension-one foliations. A structure theorem of Ghys-Gomez-MontSaludes [16] plays an important role and a version of Duminy's theorem is shown. In Section 6, the Futaki invariant is discussed. Finally, we present calculations of examples in Section 7.

I am grateful for Professor Nakagawa for his interest in this work and comments on the Futaki invariant.

\section{A Čech-de Rham theory with coefficients in $K / R$.}

1.1. Čech-de Rham cohomology. Let $K$ be either $\boldsymbol{C}$ or $\boldsymbol{R}$ and let $R$ be a discrete subgroup of $K$, where what in mind is the case where $K=C$ and $R=Z$. Let $M$ be a manifold without boundary. Fix a simple covering $\mathcal{U}=\left\{U_{i}\right\}$ of $M$ and set $U_{i_{0}, \cdots, i_{p}}=U_{i_{0}} \cap \cdots \cap U_{i_{p}}$. The Čech complex with coefficients in $R$ is denoted by $\check{C}^{*}(\mathcal{U} ; R)$, where $\check{C}^{r}(\mathcal{U} ; R)=\left\{\left\{c_{i_{0}, \cdots, i_{r}}\right\} \mid c_{i_{0}, \cdots, i_{r}} \in R\right\}$. Let $\omega=\left\{\omega_{i_{0}}, \cdots, i_{p}\right\}$ be a collection of $K$-valued $q$-forms, where each $\omega_{i_{0}}, \cdots, i_{p}$ is defined on $U_{i_{0}, \cdots, i_{p}}$. The linear space spanned by such collections is denoted by $A^{p, q}(\mathcal{U})$. There are two derivations $\delta: A^{p, q}(\mathcal{U}) \rightarrow A^{p+1, q}(\mathcal{U})$ and $d: A^{p, q}(\mathcal{U}) \rightarrow A^{p, q+1}(\mathcal{U})$. First, $d$ is just the exterior derivative. For an element $\omega=\left\{\omega_{i_{0}}, \cdots, i_{p}\right\}$ of $A^{p, q}(\mathcal{U}), \delta$ is defined by the formula

$$
(\delta \omega)_{i_{0}, \cdots, i_{p+1}}=\sum_{\alpha=0}^{p+1}(-1)^{\alpha} \omega_{i_{0}, \cdots, \hat{i_{\alpha}}, \cdots, i_{p+1}},
$$

where $\widehat{i_{\alpha}}$ means the omission. Set $\mathcal{A}_{K}^{r}(\mathcal{U})=\bigoplus_{p+q=r} A^{p, q}(\mathcal{U})$, then $\mathcal{A}_{K}^{r}(\mathcal{U})$ is equipped with a product, denoted by $\cup$, determined by requiring

$$
\left(c_{1} \cup c_{2}\right)_{i_{0} i_{1} \cdots i_{p+r}}=(-1)^{q r}\left(c_{1}\right)_{i_{0} \cdots i_{p}} \wedge\left(c_{2}\right)_{i_{p} \cdots i_{p+r}} \in A^{p+r, q+s}(\mathcal{U})
$$

for $c_{1} \in A^{p, q}(\mathcal{U})$ and $c_{2} \in A^{r, s}(\mathcal{U})$. We define mappings $D^{\prime}: A^{p, q}(\mathcal{U}) \rightarrow A^{p+1, q}(\mathcal{U})$ and 
$D^{\prime \prime}: A^{p, q}(\mathcal{U}) \rightarrow A^{p, q+1}(\mathcal{U})$ by setting $D^{\prime}=\delta$ and $D^{\prime \prime}=(-1)^{p} d$, then the equations

$$
\begin{aligned}
& D^{\prime}\left(c_{1} \cup c_{2}\right)=D^{\prime}\left(c_{1}\right) \cup c_{2}+(-1)^{p+q} c_{1} \cup\left(D^{\prime} c_{2}\right), \\
& D^{\prime \prime}\left(c_{1} \cup c_{2}\right)=D^{\prime \prime}\left(c_{1}\right) \cup c_{2}+(-1)^{p+q} c_{1} \cup\left(D^{\prime \prime} c_{2}\right),
\end{aligned}
$$

hold. We set $D=D^{\prime}+D^{\prime \prime}$, then $D$ is a derivation of $\mathcal{A}_{K}^{*}(\mathcal{U})$ such that $D^{2}=0$.

The Cech complex is naturally a subcomplex of $\mathcal{A}_{K}^{*}(\mathcal{U})$, that is, $D$ is naturally restricted to $\check{C}^{*}(\mathcal{U} ; R)$ and indeed equal to $\delta$. Its cohomology is denoted by $\check{H}^{*}(M ; R)$ and called as the Cech cohomology. On the other hand, the de Rham complex formed by $K$-valued global differential forms is also naturally a subcomplex of $\mathcal{A}_{K}^{*}(\mathcal{U})$. The cohomology is the de Rham cohomology and denoted by $H_{\mathrm{DR}}^{*}(M ; K)$.

Then the following is well-known

Theorem 1.1 (DE Rham, Weil [7]). If $K=\boldsymbol{C}$ or $K=\boldsymbol{R}$, then $H^{*}\left(\mathcal{A}_{K}^{*}(\mathcal{U}), D\right)$ is isomorphic to both $\breve{H}^{*}(M ; K)$ and $H_{\mathrm{DR}}^{*}(M ; K)$.

In order to realize $H^{*}(M ; K / R)$, the following complex is relevant.

Definition 1.2. Set

$$
\mathcal{A}_{K / R}^{*}(\mathcal{U})=\mathcal{A}_{K}^{*}(\mathcal{U}) / \check{C}^{*}(\mathcal{U} ; R)
$$

We denote again by $D$ the differential induced by the differential $D$ of $\mathcal{A}_{K}^{*}(\mathcal{U})$. Note that if we set $A_{K / R}^{p, *}=A^{p, *}(\mathcal{U})$ for $p \neq 0$ and $A_{K / R}^{0, *}=A^{0, *}(\mathcal{U}) / \check{C}^{*}(\mathcal{U} ; \boldsymbol{Z})$, then $\mathcal{A}_{K / R}^{r}(\mathcal{U})=\bigoplus_{p+q=r} A_{K / R}^{p, q}(\mathcal{U})$.

The following theorem can be shown almost in a parallel way to show Theorem 1.1 , or by chasing diagrams.

THEOREM 1.3. $H^{*}\left(\mathcal{A}_{K / R}^{*}(\mathcal{U}), D\right)$ is isomorphic to $\check{H}^{*}(M ; K / R)$.

An integration of cocycles in $\mathcal{A}^{n}(\mathcal{U})$ was introduced by Lehmann in [19], where $n=\operatorname{dim} M$. Roughly speaking, the integration is given by the sum of the integrations by pieces called 'a system of honey-comb cells' adapted to $\mathcal{U}$. Cocycles of degree other than $n$ can be integrated via Poincaré duality. The integration is known to be compatible with the usual integration of differential forms and pairing of cocycles with cycles. Moreover, the integration induces an integration of cocycles in $\mathcal{A}_{K / R}^{n}(\mathcal{U})$ valued in $K / R$. We use this integration when integrating cocycles in $\mathcal{A}_{K}^{*}(\mathcal{U})$ and $\mathcal{A}_{K / R}^{*}(\mathcal{U})$. Instead of introducing the precise definition, we give an example:

EXAMPLE 1.4. Consider $S^{1}$ as $\boldsymbol{R} / \boldsymbol{Z}$ and let $t$ be the natural coordinate. Let $U_{0}=(-\epsilon, 1 / 3+\epsilon), U_{1}=(1 / 3-\epsilon, 2 / 3+\epsilon)$ and $U_{2}=(2 / 3-\epsilon, 1+\epsilon)$ be an open covering of $S^{1}$. A system of honey-comb cells for this covering is given by setting $K_{0}=[0,1 / 3]$, $K_{1}=[1 / 3,2 / 3]$ and $K_{2}=[2 / 3,1]$. Let $\omega$ be the Cech-de Rham 1-cocycle given by the global 1 -form $d t$. Then $\omega$ is cohomologous to the cocycle $c=\left\{c_{01}, c_{12}, c_{02}\right\}$ given by $c_{01}=c_{12}=0$ and $c_{02}=2 \pi$. The integration of $\omega$ is given by

$$
\left.\int_{K_{0}} d t\right|_{U_{0}}+\left.\int_{K_{1}} d t\right|_{U_{1}}+\left.\int_{K_{2}} d t\right|_{U_{2}}=2 \pi
$$

On the other hand, the integration of $c$ is given by

$$
\int_{K_{0} \cap K_{1}} c_{01}+\int_{K_{1} \cap K_{2}} c_{12}+\int_{K_{0} \cap K_{2}} c_{02}=0+0+2 \pi=2 \pi .
$$


Note that each $K_{i} \cap K_{j}$ is a point and the integration is just summation. This is in general the case, that is, the integration restricted to $A^{\operatorname{dim} M, 0}(\mathcal{U})$ is always done by taking an appropriate sum. This is one of the most important reasons why the integration is well-defined for cocycles in $\mathcal{A}_{K / R}^{*}(\mathcal{U})$.

The support of Čech-de Rham complex is defined as follows:

Definition 1.5. Let $\omega=\left\{\omega_{i_{0}}, \cdots, i_{p}\right\}$ be a Čech-de Rham $(p, q)$-cochain. Let $I$ be the index set of the open covering $\mathcal{U}=\left\{U_{i}\right\}$ and set

$$
I_{\omega}=\left\{i \in I \mid \exists\left(i_{1}, \cdots, i_{p}\right) \in I^{p} \text { s.t. } \omega_{i, i_{1}, \cdots, i_{p}} \neq 0\right\} .
$$

The open set

$$
\operatorname{supp} \omega=\bigcup_{i \in I_{\omega}} U_{i}
$$

is called the support of $\omega$. Note that for globally defined differential forms, this support essentially coincides with the usual one by taking the covering fine enough.

1.2. Differential characters and Cheeger-Simons classes. The notion of differential characters is introduced in [8] by Cheeger and Simons as follows:

DEFinition 1.6 [8]. Let $Z_{k}$ and $B_{k}$ denote the sets of $k$-cycles and $k$-boundaries. Let $K$ be either $\boldsymbol{C}$ or $\boldsymbol{R}$, and let $R$ be a subring of $K$. Set

$$
\widehat{H}^{k}(M ; K / R)=\left\{f \in \operatorname{Hom}\left(Z_{k}, K / R\right)|f|_{B_{k}} \text { can be represented by a } k \text {-form }\right\},
$$

where a $k$-form is considered as a differential character via integration and then consider the value modulo $R$. The elements of $\widehat{H}^{k}(M ; K / R)$ is called a differential character of degree $k$ with coefficient in $K / R$.

Elements of $H^{k}(M ; K / R)$ are naturally elements of $\widehat{H}^{k}(M ; K / R)$. In particular, cocycles in $\mathcal{A}_{K / R}^{k}(\mathcal{U})$ determine elements of $\widehat{H}^{k}(M ; K / R)$ via Lehmann's integration. It is known that the mapping $H^{k}(M ; K / R) \rightarrow \widehat{H}^{k}(M ; K / R)$ is injective.

There are two important mappings $\delta_{1}: \widehat{H}^{k}(M ; K / R) \rightarrow \Lambda_{0}^{k+1}(M)$ and $\delta_{2}$ : $\hat{H}^{k}(M ; K / R) \rightarrow H^{k+1}(M, R)$, where $\Lambda_{0}^{k+1}(M)$ denotes the set of closed forms on $M$ whose periods lie in $R$. These mappings are defined as follows: let $f \in \widehat{H}^{k}(M ; K / R)$, then there is a real cochain $T$ such that $\left.\widetilde{T}\right|_{Z_{k}}=f$, where $\widetilde{T}=T \bmod R$. By assumption, there is a $(k+1)$-form $\omega$ and an $R$-valued $(k+1)$-cochain $c$ such that $T \circ \partial=\omega-c$. One can then show that $d \omega=\delta c=0$. Noticing that $T \circ \partial=\omega-c$ implies that the periods of $\omega$ lie in $R$, we set $\delta_{1}(f)=\omega$ and $\delta_{2}(f)=[c] \in H^{k+1}(M ; K / R)$. Note that if $c$ is a cocycle in the modified Cech-de Rham complex $\mathcal{A}_{K / R}^{*}(\mathcal{U}), D c \in \check{C}^{*+1}(\mathcal{U} ; R)$ and the mapping $\delta_{2}$ is given by $\delta_{2}([c])=-D c \in H^{*+1}(M ; R)$.

We cite several facts found in [8] with adaptation to our use.

Theorem 1.7. Let $\tau: H^{*}(M ; K / R) \rightarrow H^{*+1}(M ; R)$ be the connecting homomorphism, then

1) $0 \rightarrow H^{k}(M ; K / R) \rightarrow \widehat{H}^{k}(M ; K / R) \stackrel{\delta_{1}}{\rightarrow} \Lambda_{0}^{k+1}(M) \rightarrow 0$ is exact, and

2) $\delta_{2}$ restricted to $H^{k}(M ; K / R)$ coincides with $-\tau$. 
TheOREM 1.8. Let $P$ be a Chern polynomial of degree $2 k$, e.g. $P=c_{1}^{k}$, and let $\alpha=\{E, M, \nabla\}$ be a triple of a principal $\mathrm{U}(n)$-bundle $E$, the base space $M$ and $a$ connection $\nabla$. Then there is a unique element $S_{P}(\alpha) \in \widehat{H}^{2 k-1}(M ; \boldsymbol{R} / \boldsymbol{Z})$ with the following properties, namely,

1) $\delta_{1}\left(S_{P}(\alpha)\right)=P(R(\nabla))$,

2) $\delta_{2}\left(S_{P}(\alpha)\right)=P(\alpha) \in H^{2 k}(M ; Z)$,

3) $S_{P}(\alpha)$ is functorial, namely, if $\varphi: \alpha \rightarrow \alpha^{\prime}$ is a bundle morphism, then $\varphi^{*}\left(S_{P}\left(\alpha^{\prime}\right)\right)=S_{P}(\alpha)$, where $R(\nabla)$ is the curvature matrix of $\nabla$.

Corollary 1.9. Assume moreover that $P(R(\nabla))=0$ as a differential form, then $S_{P}(\alpha)$ belongs to $H^{2 k-1}(M ; \boldsymbol{R} / \boldsymbol{Z})$ and $\tau\left(S_{P}(\alpha)\right)=-P(\alpha) \in H^{2 k}(M ; Z)$.

Chern and Simons defined in [9] a differential form $T_{P}(\alpha)$ on $E$ such that $d T_{P}(\alpha)=$ $\pi^{*} P(R(\nabla))$, where $\pi: E \rightarrow M$ is the projection. If $P(R(\nabla))=0$, then $T_{P}(\alpha) \in$ $H^{2 k-1}(E ; \boldsymbol{R})$. This class is called the Chern-Simons class. These classes are related as follows:

Theorem 1.10. $\pi^{*} S_{P}(\alpha)=\widetilde{T_{P}(\alpha)} \in H^{2 k-1}(E ; \boldsymbol{R} / \boldsymbol{Z})$, where $\widetilde{T_{P}(\alpha)}$ denotes the image of $T_{P}(\alpha)$ under the mapping $H^{2 k-1}(E ; \boldsymbol{R})$ to $H^{2 k-1}(E ; \boldsymbol{R} / \boldsymbol{Z})$.

For a complex vector bundle $V$ over $M$ equipped with a connection, we set $S_{P}(V)=S_{P}(E)$ and $T_{P}(V)=T_{P}(E)$, where $E$ denotes the associate principal bundle for $V$ with the associated connection.

\section{Transversely holomorphic foliations and the Bott class.}

Definition 2.1. Let $M$ be a smooth manifold and let $\mathcal{F}=\left\{L_{\lambda}\right\}_{\lambda \in \Lambda}$ be a family of immersed submanifold of $M$ of codimension $2 q$. Let $p=\operatorname{dim} M-2 q$ and assume that $\bigcup_{\lambda \in \Lambda} L_{\lambda}=M$. The family $\mathcal{F}$ is called a transversely holomorphic foliation of complex codimension $q$ if there is an open covering $\left\{U_{i}\right\}$ of $M$ with the following properties:

1) each $U_{i}$ is homeomorphic to $V_{i} \times B_{i}$, where $V_{i}$ is an open set of $\boldsymbol{R}^{p}$ and $B_{i}$ is an open ball in $C^{q}$,

2) each connected component of $L_{\lambda} \cap U_{i}$ is of the form $V_{i} \times\{z\}, z \in B_{i}$,

3) under the identification in 1), the transition mappings are of the form $\left(x_{i}, z_{i}\right) \mapsto$ $\left(\varphi_{j i}\left(x_{i}, z_{i}, \bar{z}_{i}\right), \gamma_{j i}\left(z_{i}\right)\right)$, and $\gamma_{j i}$ is a local biholomorphic diffeomorphism.

Each $L_{\lambda}$ is called a leaf and such a covering $\left\{U_{i}\right\}$ is said to be adapted.

Definition 2.2. Let $(M, \mathcal{F})$ and $(N, \mathcal{G})$ be transversely holomorphic foliations of complex codimension $q$. A smooth mapping $f: M \rightarrow N$ is a morphism from $(M, \mathcal{F})$ to $(N, \mathcal{G})$ if $f$ maps leaves of $\mathcal{F}$ to the leaves of $\mathcal{G}$, and transversely holomorphic, that is, under the (local) identification of Definition 2.1, 2), $f$ is of the form $f(x, z)=$ $\left(f_{1}(x, z, \bar{z}), f_{2}(z)\right)$ with $f_{2}$ being holomorphic.

ExAmple 2.3. Let $E$ be a fiber bundle over a complex manifold $B$ with fiber a smooth manifold $F$, then the collection of the fibers is a transversely holomorphic foliation of $E$.

EXAMPle 2.4. (SUSPENSION) Let $M$ be a complex manifold and let $\sigma$ be a biholomorphic diffeomorphism of $M$. On $M \times \boldsymbol{R}$, there is a natural foliation by $\{z\} \times \boldsymbol{R}$, 
which is transversely holomorphic. This foliation is invariant under the mapping $(z, t) \mapsto(\sigma(z), t+1)$ and induces a natural foliation of $M_{\sigma}=M \times[0,1] /(z, 0) \sim$ $(\sigma(z), 1)$. The induced foliation $\mathcal{F}_{\sigma}$ is clearly transversely holomorphic. This type of foliations are called suspensions and used in defining the Futaki invariant (see Section $6)$.

For other constructions of examples, see [16] for instance.

The following bundles are needed for defining secondary classes.

DEFinition 2.5. Let $E$ be the complex vector subbundle of $T M \otimes C$ defined as follows. Let $\left(x_{i}, z_{i}\right)$ is a local coordinate as in Definition 2.1 (note that $x_{i}$ and $z_{i}$ are possibly not a single variable). Then one can locally speak of $\frac{\partial}{\partial x_{i}} \in T \mathcal{F} \otimes C$ and $\frac{\partial}{\partial \bar{z}_{i}} \in T M \otimes C$. As the foliation is transversely holomorphic, $\frac{\partial}{\partial \bar{z}_{i}}$ is mapped to a linear combination of $\frac{\partial}{\partial \bar{z}_{j}}$ modulo $T \mathcal{F} \otimes C$ by the transition functions. Hence the bundle locally spanned by $\frac{\partial}{\partial x_{i}}$ and $\frac{\partial}{\partial \bar{z}_{i}}$ over $C$ is well-defined, which we call $E$. The quotient bundle $Q^{1,0}(\mathcal{F})=T M \otimes C / E$ is called the complex normal bundle of $\mathcal{F}$. Finally, set $-K_{\mathcal{F}}=\bigwedge^{q} Q^{1,0}(\mathcal{F})$ and call it the anti-canonical line bundle, where $q$ is the complex codimension of $\mathcal{F}$.

Note that a morphism from $(M, \mathcal{F})$ to $(N, \mathcal{G})$ induces a bundle morphism from $Q^{1,0}(\mathcal{F})$ to $Q^{1,0}(\mathcal{G})$.

Associated with a transversely holomorphic foliation $\mathcal{F}$, there is a special kind of connections. A definition adapted to the Čech-de Rham complex is given in later, and we now give a usual definition:

DEFINITION 2.6. A connection $\nabla$ of $-K_{\mathcal{F}}$ is said to be a Bott connection if $\nabla_{X} Y=\mathcal{L}_{X} Y$ for any local sections $X$ of $E$ and $Y$ of $-K_{\mathcal{F}}$.

The notion of Bott connections is well-defined because $E$ is involutive. It is easy to see that Bott connections always exist by arguments using a partition of unity. If the complex codimension of $\mathcal{F}$ is equal to one, the above condition is rewritten as $\nabla_{X} Y=\pi[X, \widetilde{Y}]$, where $\pi: T M \otimes C \rightarrow E$ denotes the projection and $\widetilde{Y}$ is any lift of $Y$ to $T M \otimes C$.

One of the most important properties of Bott connections is the following

TheOREM 2.7 (BotT VANISHING THEOREM [6]). Let $\mathcal{F}$ be a transversely holomorphic foliation of complex codimension $q$. Let $\nabla$ be a Bott connection and $P$ be a Chern monomial of degree greater than $2 q$, e.g. $P=c_{1}^{q+1}$. Then $P(R(\nabla))$, the monomial $P$ evaluated by the curvature form $R(\nabla)$ of $\nabla$, is equal to zero as a differential form.

Classically, the Bott class is defined for the foliation with $-K_{\mathcal{F}}=0$ as follows [6]. First, let $\omega$ be a trivialization of $K_{\mathcal{F}}$, which is a 1-form such that $\operatorname{ker} \omega=$ $\left\{X \in T M \otimes C \mid \iota_{X} \omega=0\right\}=E$, where $\iota_{X}$ denotes the interior product by $X$. By the Frobenius-Nierenberg theorem, there is a 1 -form $\zeta$ such that $d \omega=-\zeta \wedge \omega$. This $\zeta$ can be seen as a Bott connection, and the differential form $(-2 \pi \sqrt{-1})^{-q-1} \zeta \wedge(d \zeta)^{q}$ is closed. The class represented by this differential form is called the Bott class and denoted by $\operatorname{Bott}_{q}(\mathcal{F})$. In the following, we explain the definition of the Bott class in 
the case where $-K_{\mathcal{F}} \neq 0$ and give a representative of $B_{q}(\mathcal{F})$ in terms of the Čech-de Rham complex.

We begin by listing the properties of the classical Bott class. Noticing that $(-2 \pi \sqrt{-1})^{-1} d \zeta$ represents the image of $c_{1}\left(-K_{\mathcal{F}}\right)$ in $H^{2}(M ; C)$, we see that the Bott class is the pull-back of the Chern-Simons class $T_{c_{1}^{q+1}}\left(-K_{\mathcal{F}}\right)$ by the trivialization, where $-K_{\mathcal{F}}$ is equipped with any Bott connection. It is known that the imaginary part of the Bott class is well-defined even if $-K_{\mathcal{F}} \neq 0$, that is, there is a well-defined element $I B_{q}(\mathcal{F})$ of $H^{2 q+1}(M ; R)$ such that $I B_{q}(\mathcal{F})=\operatorname{ImBott}_{q}(\mathcal{F})$ if $-K_{\mathcal{F}}=0$. A definition of $I B_{q}(\mathcal{F})$ will be given in Section 4 (Theorem-Definition 4.2).

In view of these facts, the Bott class is now defined as follows (cf. [13]):

Definition 2.8. The Bott class is the Cheeger-Simons class $S_{c_{1}^{q+1}}\left(-K_{\mathcal{F}}\right)$ for $c_{1}^{q+1}$ of $-K_{\mathcal{F}}$ equipped with any Bott connection. It is an element of $H^{2 q+1}(M ; \boldsymbol{C} / \boldsymbol{Z})$ and denoted by $B_{q}(\mathcal{F})$. Equivalently, $B_{q}(\mathcal{F})$ is the unique element of $H^{2 q+1}(M ; C / Z)$ with the following properties:

1) The real part of $B_{q}(\mathcal{F})$ is the Cheeger-Simons class for $c_{1}^{q+1}$ of $-K_{\mathcal{F}}$ equipped with any Bott connection. It is an element of $H^{2 q+1}(M ; \boldsymbol{R} / \boldsymbol{Z})$ which satisfies $\tau\left(B_{q}(\mathcal{F})\right)=$ $-c_{1}\left(-K_{\mathcal{F}}\right)^{q+1}$ and which is functorial under the morphism between transversely holomorphic foliations, where $\tau$ is the connecting homomorphism as in Theorem 1.7.

2) The imaginary part of $B_{q}(\mathcal{F})$ is equal to $I B_{q}(\mathcal{F})$.

It is well-known that $B_{q}(\mathcal{F})$ is independent of the choice of Bott connection. This fact will been shown in a more precise version in Lemma 2.16.

In general $-K_{\mathcal{F}} \neq 0$ and the class $c_{1}^{q+1}\left(-K_{\mathcal{F}}\right)$ can have a torsion part. The following example is a modification of the ones found in [17] and [22].

EXAMPLE 2.9. Let $k$ be an integer greater than 2 and denote $\boldsymbol{Z}_{m}$ by $\boldsymbol{Z} / m \boldsymbol{Z}$,

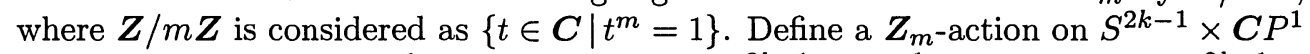
by $t\left(x,\left[z_{0}: z_{1}\right]\right)=\left(t x,\left[t^{-1} z_{0}: z_{1}\right]\right)$. Let $M=\left(S^{2 k-1} \times C P^{1}\right) / Z_{m}$ and $U=\left(S^{2 k-1} \times\right.$ $\boldsymbol{C}) / \boldsymbol{Z}_{m}$, where $\boldsymbol{C}=\{[z: 1]\} \subset \boldsymbol{C} P^{1}$, then $M$ is naturally equipped with a foliation whose leaves are $\left(S^{2 k-1} \times\left\{\left[z_{0}: z_{1}\right]\right\}\right) / Z_{m}$. We denote this foliation by $\mathcal{F}$, then $\mathcal{F}$ is naturally restricted to $U$. Note that $U$ is a line bundle over the Lens space $L(m ; 1)=S^{2 k-1} / \boldsymbol{Z}_{m}$ and that it is isomorphic to the complex normal bundle of $\mathcal{F}$ pulled back by the inclusion of $L(m ; 1) \times\{[0: 1]\}$ into $M$. On the other hand, the projection from $M$ to $L(m ; 1)$ induces an injection from $H^{*}(U ; \boldsymbol{Z}) \cong H^{*}(L(m ; 1) ; \boldsymbol{Z})$ to $H^{*}(M ; \boldsymbol{Z})$. Hence it suffices to see that $c_{1}(U)^{2}$ has a torsion part. This is seen as follows. First notice that $[x, z] \rightarrow([x], z x)$ gives the embedding of $U$ to $L(m ; 1) \times C^{k}$, where the bracket means the equivalence class. It follows that $U$ is the pull-back of the tautological bundle over $C P^{k-1}$ by the natural projection, which we denote by $\pi$. As $\pi^{*}$ is the projection from $Z$ to $Z / m Z$ in degree $4, c_{1}(U)^{2}$ is its generator.

Now we begin to write down the Bott class in terms of the Cech-de Rham complex. We adopt the following

Notation 2.10.

1) A Čech-de Rham cochain is expressed by omitting indices of local forms, for example, the cochain $\left\{\zeta_{i}\right\}$ is denoted by $\zeta$ and vice versa.

2) If $f$ is a Čech-de Rham $(p, 0)$-cochain, namely, a family of locally defined functions, we denote by $\log f$ the $(p, 0)$-cochain defined by $(\log f)_{i_{0}, \cdots, i_{p}}=\log \left(f_{i_{0}, \cdots, i_{p}}\right)$. 
Here we assume that $f_{i_{0}, \cdots, i_{p}}$ is non-zero and that its logarithm is well-defined by taking refinements of covering and choosing a branch.

Let $\mathcal{U}=\left\{U_{i}\right\}$ be an adapted covering. Assume that $-K_{\mathcal{F}}$ is trivial when restricted to each $U_{i}$ and let $e_{i}$ be a trivialization on $U_{i}$. Let $\left\{\widetilde{\alpha}_{i j}\right\}$ be a family of non-zero functions such that $e_{i}=\widetilde{\alpha}_{i j} e_{j}$. Let $\nabla_{i}$ be a Bott connection defined on $U_{i}$ and let $\widetilde{\zeta}_{i}$ be its connection form with respect to $e_{i}$. Write $\widetilde{\zeta}_{j}-\widetilde{\zeta}_{i}=-\frac{d \widetilde{\alpha}_{i j}}{\widetilde{\alpha}_{i j}}+\widetilde{\beta}_{i j}$, then each $\widetilde{\beta}_{i j}$ is of transverse type- $(1,0)$, that is, $\widetilde{\beta}_{i j}$ is written in terms of $d z_{j}^{k}$.

We use the following normalized cochains.

DEFINITION 2.11. Let $\widetilde{\zeta}_{i}, \widetilde{\alpha}_{i j}$ and $\widetilde{\beta}_{i j}$ as above. Consider them as Čech-de Rham cochains and define other cochains as follows:

$$
\begin{aligned}
\zeta & =\frac{-1}{2 \pi \sqrt{-1}} \widetilde{\zeta} \\
\log \alpha & =\frac{-1}{2 \pi \sqrt{-1}} \log \widetilde{\alpha} \\
\frac{d \alpha}{\alpha} & =\frac{-1}{2 \pi \sqrt{-1}} \frac{d \widetilde{\alpha}}{\widetilde{\alpha}}, \\
\beta & =\frac{-1}{2 \pi \sqrt{-1}} \widetilde{\beta} \\
\Theta & =\frac{-1}{2 \pi \sqrt{-1}} \delta \log \widetilde{\alpha}=\delta \log \alpha .
\end{aligned}
$$

Note that $d \log \alpha=\frac{d \alpha}{\alpha}$ as cochains. Note also that $\Theta$ is a Čech 2-cocycle which represents $c_{1}\left(-K_{\mathcal{F}}\right)$ in $\breve{H}^{2}(M ; Z)$ by definition.

The following properties of these cochains are useful.

LEMMA 2.12.

1) $d \zeta_{i}$ is a linear combination of the differential forms such as $\omega \wedge d z_{i}^{k}$, where $\omega$ is some 1-form.

2) The following equations hold:

$$
\begin{aligned}
& \delta \zeta=-\frac{d \alpha}{\alpha}+\beta \\
& \delta\left(\frac{d \alpha}{\alpha}\right)=\delta \beta=0 \\
& \delta d \zeta=d \delta \zeta=d \beta
\end{aligned}
$$

3) The cochain $d \zeta+\beta$ is cohomologous to $\Theta$. More precisely, $D(\zeta-\log \alpha)=$ $d \zeta+\beta-\Theta$.

Proof. The first part follows directly from the definition of the Bott connection (cf. [6]). The part 2) follows from straightforward calculations. The last claim is also shown by a simple calculation. We present it for explaining how differential of 
cochains are computed. By definition $D^{\prime \prime}(\log \alpha)=-d \log \alpha$. Hence

$$
\begin{aligned}
D(\zeta-\log \alpha) & =-\frac{d \alpha}{\alpha}+\beta+d \zeta-\delta \log \alpha+\frac{d \alpha}{\alpha} \\
& =d \zeta+\beta-\Theta .
\end{aligned}
$$

The following cocycle will represent the Bott class.

Definition 2.13. Let $\nabla=\left\{\nabla_{i}\right\}$ be a family of local Bott connections and $e=\left\{e_{i}\right\}$ be a family of local trivializations as above. We define a cochain $B_{q}(\nabla, e)$ in $\mathcal{A}_{C}^{*}(\mathcal{U})$ by the formula

$$
\begin{aligned}
B_{q}(\nabla, e)= & (\zeta-\log \alpha) \cup(d \zeta+\beta)^{q} \\
& +\Theta \cup(\zeta-\log \alpha) \cup(d \zeta+\beta)^{q-1} \\
& +\cdots+\Theta^{q} \cup(\zeta-\log \alpha) .
\end{aligned}
$$

The image of $B_{q}(\nabla, e)$ in $\mathcal{A}_{C / \boldsymbol{Z}}^{*}(\mathcal{U})$ is also denoted by $B_{q}(\nabla, e)$.

We will show that $B_{q}(\nabla, e)$ indeed represents the Bott class in $H^{2 q+1}(M ; C / Z)$. The following cochain is useful for this purpose:

Definition 2.14. Let $\Omega$ and $\Omega^{\prime}$ be cocycles in $\mathcal{A}_{C}^{2 m}(\mathcal{U})$ and let $\omega$ be a cochain in $\mathcal{A}_{C}^{2 m-1}(\mathcal{U})$ such that $D \omega=\Omega^{\prime}-\Omega$. We set

$$
\Delta_{\omega}\left(\Omega^{\prime k}, \Omega^{k}\right)= \begin{cases}\omega \cup \Omega^{\prime k-1}+\Omega \cup \omega \cup \Omega^{\prime k-2}+\cdots+\Omega^{k-1} \cup \omega & \text { if } k>1 \\ \omega & \text { if } k=1\end{cases}
$$

LEMMA 2.15. The cochian $\Delta_{\omega}\left(\Omega^{\prime k}, \Omega^{k}\right)$ has the following properties:

1) $D \Delta_{\omega}\left(\Omega^{\prime k}, \Omega^{k}\right)=\Omega^{\prime k}-\Omega^{k}$.

2) $\Delta_{\omega}\left(\Omega^{\prime k+1}, \Omega^{k+1}\right)=\Delta_{\omega}\left(\Omega^{\prime k}, \Omega^{k}\right) \cup \Omega^{\prime}+\Omega^{k} \cup \omega=\omega \cup \Omega^{\prime k}+\Omega \cup \Delta_{\omega}\left(\Omega^{\prime k}, \Omega^{k}\right)$.

The proof is straightforward and omitted. We now show the following

LEMMA 2.16. The cochain $B_{q}(\nabla, e)$ has the following properties:

1) $D B_{q}(\nabla, e)=-\Theta^{q+1}$ in $\mathcal{A}_{C}^{*}(\mathcal{U})$.

2) Let $e$ and $e^{\prime}$ be families of local trivializations. Define cochains $\zeta, d \zeta, \log \alpha$, $\beta$ and $\Theta$ by using $e$ and $\zeta^{\prime}, d \zeta^{\prime}, \log \alpha^{\prime}, \beta^{\prime}$ and $\Theta^{\prime}$ by $e^{\prime}$. Let $g=\left\{g_{i}\right\}$ be the family of non-zero functions satisfying $e_{i}^{\prime}=g_{i} e_{i}$. We assume by taking refinements of coverings that each $\log g_{i}$ is well-defined.

Under these assumptions, there is a cochain $\nu \in \check{C}^{*}(\mathcal{U} ; Z)$ such that $\Theta^{\prime}-\Theta=D \nu$, and if we set

$$
\begin{aligned}
& \sigma_{i}=(-2 \pi \sqrt{-1})^{-1} \Theta^{i} \cup(\log g) \cup(d \zeta+\beta)^{q-i}, \\
& \rho_{k}=\Delta_{\nu}\left(\Theta^{\prime k+1}, \Theta^{k+1}\right) \cup\left(\zeta^{\prime}-\log \alpha^{\prime}\right) \cup(d \zeta+\beta)^{q-k-1},
\end{aligned}
$$

for $i=0, \cdots, q$ and $k=0,1, \cdots, q-1$, then

$$
B_{q}\left(\nabla, e^{\prime}\right)-B_{q}(\nabla, e)=\sum_{i=0}^{q} D \sigma_{i}+\sum_{k=0}^{q-1} D \rho_{k}
$$

holds in $\mathcal{A}_{\boldsymbol{C} / \boldsymbol{Z}}^{*}(\mathcal{U})$. 
3) Let $\nabla=\left\{\zeta_{i}\right\}$ and $\nabla^{\prime}=\left\{\zeta_{i}^{\prime}\right\}$ be families of local Bott connections and let $\beta$, $\beta^{\prime}, d \zeta$ and $d \zeta^{\prime}$ be corresponding cochains.

Define a cochain $\gamma=\left\{\gamma_{i}\right\}$ by setting $\gamma_{i}=\zeta_{i}^{\prime}-\zeta_{i}$, then $D \gamma=\left(d \zeta^{\prime}+\beta^{\prime}\right)-(d \zeta+\beta)$. If we set, for $k=0, \cdots, q-1$,

$$
\omega_{k}=\Theta^{k} \cup\left(\zeta^{\prime}-\log \alpha\right) \cup \Delta_{-\gamma}\left((d \zeta+\beta)^{q-k},\left(d \zeta^{\prime}+\beta^{\prime}\right)^{q-k}\right),
$$

then $B_{q}\left(\nabla^{\prime}, e\right)-B_{q}(\nabla, e)=D \omega_{0}+\cdots+D \omega_{q-1}$ as cocycles in $\mathcal{A}_{C}^{*}(\mathcal{U})$.

Hence the cocycle $B_{q}(\nabla, e)$ represents a cohomology class in $H^{2 q+1}(M ; C / Z)$ which is independent of $\nabla$ and $e$.

REMARK 2.17. The independence of the class can be deduced from general properties of Cheeger-Simons classes without writing down the cochains such as $\sigma_{i}$, $\rho_{k}$ and $\omega_{k}$ (cf. Proposition 7.3 of [8]), but their explicit forms are needed for defining the residue in the next section. More precisely, we need the following properties: first, the support of $\omega_{k}$ in 3) is contained in a neighborhood of the set where $\nabla^{\prime} \neq \nabla$, because $\gamma=0$ if $\nabla^{\prime}=\nabla$. Second, the support of $\sigma_{i}$ and $\rho_{k}$ in 2) is contained in a neighborhood of the set where $e \neq e^{\prime}$.

Proof of Lemma 2.16. Since $D(\zeta-\log \alpha)=(d \zeta+\beta)-\Theta$ by Lemma 2.12, the cochain $B_{q}(\nabla, e)$ is equal to $\Delta_{(\zeta-\log \alpha)}\left((d \zeta+\beta)^{q+1}, \Theta^{q+1}\right)$. As $(d \zeta+\beta)^{q+1}=0$ by the degree reason in the transversal direction, $D B_{q}(\nabla, e)=-\Theta^{q+1}$.

Now we show 2). Let $e^{\prime}$ be another family of local trivializations and define cochains as in the statement. These cochains are related as follows. First, $\widetilde{\alpha}_{i j}=$ $g_{i}^{-1} \widetilde{\alpha}_{i j}^{\prime} g_{j}$. Hence there is a family $\nu=\left\{\nu_{i j}\right\}$ of integers such that $\log \widetilde{\alpha}^{\prime}-\log \widetilde{\alpha}=$ $-\delta \log g+(-2 \pi \sqrt{-1}) \nu$. Dividing the both hand sides by $(-2 \pi \sqrt{-1})$, we see that $\log \alpha^{\prime}-\log \alpha=-(-2 \pi \sqrt{-1})^{-1} \delta \log g+\nu$. On the other hand, $\zeta_{i}^{\prime}=\zeta_{i}+\frac{-1}{2 \pi \sqrt{-1}} \frac{d g_{i}}{g_{i}}$ and $d \zeta^{\prime}=d \zeta$. It follows from these relations that $\beta^{\prime}=\beta, \Theta^{\prime}=\Theta+\delta \nu$ and $\zeta^{\prime}-\zeta-$ $\log \alpha^{\prime}+\log \alpha=(-2 \pi \sqrt{-1})^{-1} D \log g-\nu$. We now have the following equation, namely,

$$
\begin{aligned}
& B_{q}\left(\nabla, e^{\prime}\right)-B_{q}(\nabla, e) \\
= & \left((-2 \pi \sqrt{-1})^{-1} D \log g-\nu\right) \cup(d \zeta+\beta)^{q} \\
& +\Theta \cup\left((-2 \pi \sqrt{-1})^{-1} D \log g-\nu\right) \cup(d \zeta+\beta)^{q-1} \\
& +\left(\Theta^{\prime}-\Theta\right) \cup\left(\zeta^{\prime}-\log \alpha^{\prime}\right) \cup(d \zeta+\beta)^{q-1} \\
& +\Theta^{2} \cup\left((-2 \pi \sqrt{-1})^{-1} D \log g-\nu\right) \cup(d \zeta+\beta)^{q-2} \\
& +\left(\Theta^{\prime 2}-\Theta^{2}\right) \cup\left(\zeta^{\prime}-\log \alpha^{\prime}\right) \cup(d \zeta+\beta)^{q-2} \\
& +\cdots+\Theta^{q} \cup\left((-2 \pi \sqrt{-1})^{-1} D \log g-\nu\right) \\
& +\cdots+\left(\Theta^{\prime}-\Theta^{q}\right) \cup\left(\zeta^{\prime}-\log \alpha^{\prime}\right) .
\end{aligned}
$$

On the other hand, as $d \zeta^{\prime}+\beta^{\prime}=d \zeta+\beta$,

$$
\begin{aligned}
D \rho_{k}= & \left(\Theta^{\prime k+1}-\Theta^{k+1}\right) \cup\left(\zeta^{\prime}-\log \alpha^{\prime}\right) \cup(d \zeta+\beta)^{q-k-1} \\
& -\Delta_{\nu}\left(\Theta^{\prime k+1}, \Theta^{k+1}\right) \cup(d \zeta+\beta)^{q-k} \\
& +\Delta_{\nu}\left(\Theta^{\prime k+1}, \Theta^{k+1}\right) \cup \Theta^{\prime} \cup(d \zeta+\beta)^{q-k-1}
\end{aligned}
$$


Hence, by 2) of Lemma 2.15 ,

$$
B_{q}\left(\nabla, e^{\prime}\right)-B_{q}(\nabla, e)-\sum_{i=0}^{q} D \sigma_{i}-\sum_{k=0}^{q-1} D \rho_{k}=\Delta_{\nu}\left(\Theta^{\prime q+1}, \Theta^{q+1}\right) .
$$

The right hand side belongs to $\check{C}^{*}(\mathcal{U} ; Z)$ because it is a combination of $\nu, \Theta^{\prime}$ and $\Theta$. This completes the proof of the part 2).

Finally we show the part 3). Fix a family of local trivializations $e=\left\{e_{i}\right\}$ and let $\nabla^{\prime}$ be another family of local Bott connections, and define cochains as in the statement. As $\nabla$ and $\nabla^{\prime}$ are Bott connections, $\gamma_{i}$ is of transverse type- $(1,0)$. By comparing cochains, we see that $\beta^{\prime}=\beta+\delta \gamma$ and $D \gamma=d \zeta^{\prime}-d \zeta+\beta^{\prime}-\beta$. On the other hand, we have the following equation:

$$
\begin{aligned}
D \omega_{k}= & -\Theta^{k+1} \cup \Delta_{-\gamma}\left((d \zeta+\beta)^{q-k},\left(d \zeta^{\prime}+\beta^{\prime}\right)^{q-k}\right) \\
& +\Theta^{k} \cup\left(d \zeta^{\prime}+\beta^{\prime}\right) \cup \Delta_{-\gamma}\left((d \zeta+\beta)^{q-k},\left(d \zeta^{\prime}+\beta^{\prime}\right)^{q-k}\right) \\
& -\Theta^{k} \cup\left(\zeta^{\prime}-\log \alpha\right) \cup\left((d \zeta+\beta)^{q-k}-\left(d \zeta^{\prime}+\beta^{\prime}\right)^{q-k}\right)
\end{aligned}
$$

Noticing that $\left(d \zeta^{\prime}+\beta^{\prime}\right)^{k} \cup \gamma \cup(d \zeta+\beta)^{q-k}=0$, we see that $B_{q}\left(\nabla^{\prime}, e\right)-B_{q}(\nabla, e)=$ $D \omega_{0}+\cdots+D \omega_{q-1}$. This completes the proof.

Definition 2.18. The cocycle $B_{q}(\nabla, e)$ is called the Bott cocycle with respect $\nabla$ and $e$.

THEOREM 2.19. The Bott cocycle represents the Bott class in $H^{2 q+1}(M ; C / Z)$. That is, if we denote by $B$ the class represented by the Bott cocycle, then

1) The real part of $B$ is the Cheeger-Simons class for $c_{1}^{q+1}$ of $-K_{\mathcal{F}}$ equipped with any Bott connection.

2) The imaginary part of $B$ coincides with $I B_{q}(\mathcal{F})$. Moreover, if $-K_{\mathcal{F}}=0$, then $B_{q}(\mathcal{F})=\operatorname{Bott}_{q}(\mathcal{F}) \bmod \boldsymbol{Z}$.

Proof. The first claim is a direct consequence of Lemma 2.16. In order to show the second claim, we compute $B_{q}(\nabla, e)-\overline{B_{q}(\nabla, e)}$ for a suitable choice of $\nabla$ and $e$. First write $e_{i}=f_{i} \frac{\partial}{\partial z_{i}^{1}} \wedge \cdots \wedge \frac{\partial}{\partial z_{i}^{q}}$. As $-K_{\mathcal{F}} \otimes\left(-\overline{K_{\mathcal{F}}}\right)$ is always trivial, we may assume that $\left|f_{j}\right|^{2}=\left|\left(f_{i} \circ \varphi_{i j}\right) \operatorname{det} \gamma_{i j}^{\prime}\right|^{2}$. Hence each $f_{i}$ can be assumed to be a positive real valued function and we may assume that $f_{j}=\left(f_{i} \circ \varphi_{i j}\right)\left|\operatorname{det} \gamma_{i j}^{\prime}\right|$. It follows by definition that $\tilde{\alpha}_{i j}=\frac{\operatorname{det} \gamma_{i j}^{\prime}}{\left|\operatorname{det} \gamma_{i j}^{\prime}\right|}$. We may also assume that $\nabla$ is a global Bott connection. Then $\beta=0$ and $\delta \zeta=-\frac{d \alpha}{\alpha}$. Note that $\log \alpha-\overline{\log \alpha}=0, \delta(\zeta-\bar{\zeta})=0$ and $\delta(d \zeta)=\delta(d \bar{\zeta})=0$, where the sign is due to the normalization we made. Note also that

$$
\begin{aligned}
D(\zeta \cup \bar{\zeta}) & =-\frac{d \alpha}{\alpha} \cup \bar{\zeta}+\zeta \cup \frac{d \alpha}{\alpha}+d \zeta \cup \bar{\zeta}-\zeta \cup d \bar{\zeta} \\
& =\frac{d \alpha}{\alpha} \cup(\zeta-\bar{\zeta})+\bar{\zeta} \cup d \zeta-\zeta \cup d \bar{\zeta},
\end{aligned}
$$

because

$$
\left(\frac{d \alpha}{\alpha} \cup \zeta-\zeta \cup \frac{d \alpha}{\alpha}\right)_{i j}=\frac{d \alpha_{i j}}{\alpha_{i j}} \wedge \zeta_{j}+\zeta_{i} \wedge \frac{d \alpha_{i j}}{\alpha_{i j}}=\frac{d \alpha_{i j}}{\alpha_{i j}} \wedge\left(\zeta_{j}-\zeta_{i}\right)=-\frac{d \alpha_{i j}}{\alpha_{i j}} \wedge \frac{d \alpha_{i j}}{\alpha_{i j}}=0
$$


Let $I_{k}$ and $J_{k}$ be the cochains defined by

$$
\begin{aligned}
I_{k}= & \Theta^{q} \cup(\zeta-\log \alpha)-\Theta^{q} \cup(\bar{\zeta}-\overline{\log \alpha}) \\
& +\Theta^{q-1} \cup(\zeta-\log \alpha) \cup d \zeta-\Theta^{q-1} \cup(\bar{\zeta}-\overline{\log \alpha}) \cup d \bar{\zeta} \\
& +\cdots \\
& +\Theta^{q-k} \cup(\zeta-\log \alpha) \cup(d \zeta)^{k}-\Theta^{q-k} \cup(\bar{\zeta}-\overline{\log \alpha}) \cup(d \bar{\zeta})^{k} \\
J_{k}= & \Theta^{q-k} \cup(\zeta-\bar{\zeta}) \cup\left((d \zeta)^{k}+(d \zeta)^{k-1} \cup d \bar{\zeta}+\cdots+(d \bar{\zeta})^{k}\right) .
\end{aligned}
$$

It suffices to show that $I_{k}$ and $J_{k}$ are cohomologous, because $I_{q}=B_{q}(\nabla, e)-\overline{B_{q}(\nabla, e)}$ and $J_{q}=-2 I B_{q}(\mathcal{F})$ (see Definition 4.1 and Theorem-Definition 4.2). Here we used the fact that $\eta+\bar{\eta}=0$ under our choice of $\alpha$.

The claim is shown as follows. First,

$$
\begin{aligned}
I_{0} & =\Theta^{q} \cup(\zeta-\log \alpha)-\Theta^{q} \cup(\bar{\zeta}-\overline{\log \alpha}) \\
& =\Theta^{q} \cup(\zeta-\bar{\zeta}) \\
& =J_{0}
\end{aligned}
$$

Now set

$$
\begin{aligned}
(d \zeta, d \bar{\zeta})^{k}= & (d \zeta)^{k}+(d \zeta)^{k-1} \cup(d \bar{\zeta})+\cdots+(d \bar{\zeta})^{k}, \text { and } \\
\omega_{k}= & -\Theta^{q-k} \cup \log \alpha \cup(\zeta-\bar{\zeta}) \cup(d \zeta, d \bar{\zeta})^{k-1} \\
& +\Theta^{q-k} \cup \zeta \cup(-\bar{\zeta}) \cup(d \zeta, d \bar{\zeta})^{k-1},
\end{aligned}
$$

then

$$
\begin{aligned}
D \omega_{k}= & -\Theta^{q-k+1} \cup(\zeta-\bar{\zeta}) \cup(d \zeta, d \bar{\zeta})^{k-1} \\
& +\Theta^{q-k} \cup \frac{d \alpha}{\alpha} \cup(\zeta-\bar{\zeta}) \cup(d \zeta, d \bar{\zeta})^{k-1} \\
& +\Theta^{q-k} \cup \log \alpha \cup\left((d \zeta)^{k}-(d \bar{\zeta})^{k}\right) \\
& +\Theta^{q-k} \cup\left(-\frac{d \alpha}{\alpha}\right) \cup(\zeta-\bar{\zeta}) \cup(d \zeta, d \bar{\zeta})^{k-1} \\
& +\Theta^{q-k} \cup(-\bar{\zeta} \cup d \zeta+\zeta \cup d \bar{\zeta}) \cup(d \zeta, d \bar{\zeta})^{k-1} \\
= & -\Theta^{q-k+1} \cup(\zeta-\bar{\zeta}) \cup(d \zeta, d \bar{\zeta})^{k-1} \\
& +\Theta^{q-k} \cup \log \alpha \cup\left((d \zeta)^{k}-(d \bar{\zeta})^{k}\right) \\
& -\Theta^{q-k} \cup(\bar{\zeta} \cup d \zeta-\zeta \cup d \bar{\zeta}) \cup(d \zeta, d \bar{\zeta})^{k-1}
\end{aligned}
$$

On the other hand,

$$
\begin{aligned}
& \Theta^{q-k} \cup(\zeta-\log \alpha) \cup(d \zeta)^{k}-\Theta^{q-k} \cup(\bar{\zeta}-\overline{\log \alpha}) \cup(d \bar{\zeta})^{k} \\
= & \Theta^{q-k} \cup\left(\zeta \cup(d \zeta)^{k}-\bar{\zeta} \cup(d \bar{\zeta})^{k}\right) \\
& -\Theta^{q-k} \cup \log \alpha \cup\left((d \zeta)^{k}-(d \bar{\zeta})^{k}\right) .
\end{aligned}
$$

It is straightforward to see that

$$
(-\bar{\zeta} \cup d \zeta+\zeta \cup d \bar{\zeta}) \cup(d \zeta, d \bar{\zeta})^{k-1}+\zeta \cup(d \zeta)^{k}-\bar{\zeta} \cup(d \bar{\zeta})^{k}=(\zeta-\bar{\zeta}) \cup(d \zeta, d \bar{\zeta})^{k}
$$


Hence $I_{k}+D \omega_{1}+\cdots+D \omega_{k}=\Theta^{q-k} \cup(\zeta-\bar{\zeta}) \cup(d \zeta, d \bar{\zeta})^{k}$. This completes the proof of 2).

Finally, assume that $-K_{\mathcal{F}}=0$, then choosing $e$ as a global trivialization, we may assume that $\log \alpha=0$. On the other hand, assuming that $\nabla$ is a global Bott connection, $\beta=0$. Then $B_{q}(\nabla, e)=\zeta \cup(d \zeta)^{q}$. Taking the normalization into account, the proof is completed.

It is worth mentioning that there is a natural choice of families of local connections and local trivializations. Let $e_{i}=\frac{\partial}{\partial z_{i}^{1}} \wedge \cdots \wedge \frac{\partial}{\partial z_{i}^{q}}$, then $\widetilde{\alpha}_{i j}=\operatorname{det} \gamma_{i j}^{\prime}$. Now choose $0_{i}=0$ as a local Bott connection: $\zeta=\left\{0_{i}\right\}$, then $\beta$ is by definition given by $\beta_{i j}=$ $\frac{-1}{2 \pi \sqrt{-1}} \frac{d \alpha_{i j}}{\alpha_{i j}}=\frac{-1}{2 \pi \sqrt{-1}} d \log \operatorname{det} \gamma_{i j}^{\prime}$. Thus we obtained the following:

Corollary 2.20. The Bott class $B_{q}(\mathcal{F})$ is represented by the cocycle

$$
\begin{aligned}
& -(-2 \pi \sqrt{-1})^{-q-1} \log \operatorname{det} \gamma_{i j}^{\prime} \cup\left(d \log \operatorname{det} \gamma_{i j}^{\prime}\right)^{q} \\
& -(-2 \pi \sqrt{-1})^{-q-1}\left(\delta \log \operatorname{det} \gamma_{i j}^{\prime}\right) \cup\left(\log \operatorname{det} \gamma_{i j}^{\prime}\right) \cup\left(d \log \operatorname{det} \gamma_{i j}^{\prime}\right)^{q-1} \\
& -\cdots-(-2 \pi \sqrt{-1})^{-q-1}\left(\delta \log \operatorname{det} \gamma_{i j}^{\prime}\right)^{q} \cup\left(\log \operatorname{det} \gamma_{i j}^{\prime}\right) .
\end{aligned}
$$

REMARK 2.21. The imaginary part of the above formula has already appeared in $[1]$.

3. Residue. A residue of the Bott class together with the notion of $\Gamma$-vector field is first introduced by Heitsch [18]. They can be generalized as follows.

Definition 3.1. A vector field $X$ is said to be a $\Gamma$-vector field for $\mathcal{F}$ if $X$ is defined on an open set, say $O_{X}$, and $[E, X] \subset E$ on $O_{X}$. This condition implies that $\mathcal{F}$ and $X$ form a transversely holomorphic foliation of complex codimension $q-1$ on the open set $M \backslash Z_{X}$, where $Z_{X}=\{X \in E\} \cup\left(M \backslash O_{X}\right)$. We denote this foliation by $\mathcal{F}_{X}$. Note that $Z_{X}$ is saturated by leaves of $\mathcal{F}$ if $O_{X}$ is also.

In terms of the local coordinates as in Definition 2.1, the above condition is rephrased as follows: let $(x, z)=\left(\left(x^{1}, \cdots, x^{p}\right),\left(z^{1}, \cdots, z^{q}\right)\right)$ be a local coordinate as in Definition 2.1, then $X$ is a $\Gamma$-vector field for $\mathcal{F}$ if $X$ is locally of the form $\sum f_{j}(x, z, \bar{z}) \frac{\partial}{\partial x^{j}}+\sum g_{a}(z) \frac{\partial}{\partial z^{a}}+\sum k_{b}(x, z, \bar{z}) \frac{\partial}{\partial \bar{z}^{b}}$ with $g_{a}$ 's being holomorphic, and $Z_{X}=\{X=0$ when projected to $Q(\mathcal{F})\} \cup\left(M \backslash O_{X}\right)$.

Definition 3.2. Let $X$ be a $\Gamma$-vector field for $\mathcal{F}$. A Bott connection $\nabla$ of $-K_{\mathcal{F}}$ is said to be a basic $X$-connection for $\mathcal{F}$ supported off $U$ if $\nabla$ is a Bott connection for $\mathcal{F}_{X}$ on a neighborhood $U$ of $M \backslash Z_{X}$. In particular, $\nabla_{X} s=\mathcal{L}_{X} s$ on $U$, where $\mathcal{L}_{X}$ denotes the Lie derivative by $X$. Basic $X$-connections are usually denoted by $\nabla^{X}$.

Note that we may assume, if necessary, that $\nabla^{X}$ is globally defined by using a partition of unity. Then it is a basic $X$-connection for $\mathcal{F}$ supported off $U$ in the sense of Heitsch [18].

Let $X$ be a $\Gamma$-vector field for $\mathcal{F}$ and let $U_{X}$ be an open neighborhood of $Z_{X}$. Suppose that there is an open neighborhood $U$ of $M \backslash U_{X}$ on which a trivialization $e_{U}$ of $-K_{\mathcal{F}}$ exists. Let $\mathcal{U}$ be an open covering of $M$ which is a refinement of $\left\{U, U_{X}\right\}$. Now let $\nabla^{X}$ be a basic $X$-connection supported off $U$ and let $e=\left\{e_{i}\right\}$ be a family 
of local trivializations such that $e_{i}=e_{U}$ if $U_{i} \subset U$. Then, $B_{q}\left(\nabla^{X}, e\right)=0$ as cocycles on $U$ because the cochains $\log \alpha$ and $\Theta$ are zero on $U$ and $(d \zeta+\beta)^{q}=0$ by virtue of the Bott vanishing theorem for $\mathcal{F}_{X}$. Therefore, the cocycle $B_{q}\left(\nabla^{X}, e\right)$ determines an element of $H^{2 q+1}\left(M, M \backslash U_{X} ; C / Z\right)$, or $H_{c}^{2 q+1}\left(U_{X} ; C / Z\right)$.

Definition 3.3. Let $X$ be a basic $\Gamma$-vector field for $\mathcal{F}, e_{U}$ be a trivialization of $-K_{\mathcal{F}}$ on a neighborhood $U$ on $M \backslash U_{X}$. Let $\nabla^{X}$ be a basic- $X$ connection for $\mathcal{F}$ supported off $U$ and $e$ be a family of local trivialization as above. We set

$$
\operatorname{res} B_{q}(\mathcal{F}, X, e)=\left[B_{q}\left(\nabla^{X}, e\right)\right] \in H_{c}^{2 q+1}\left(U_{X} ; C / Z\right)
$$

and call it the residue of the Bott class at $U_{X}$ with respect to $X$ and $e$.

The residue has the same properties as the original one:

TheOREm 3.4. The residue $\operatorname{res} B_{q}(\mathcal{F}, X, e)$ is independent of the choice of $\nabla^{X}$. Precisely speaking, the residue is determined by $X$, the restriction of $\mathcal{F}$ to $U_{X}$ and the homotopy type of e on $U$. If $U_{X}=U_{1} \cup \cdots \cup U_{r}$ is the decomposition of $U_{X}$ into its components, then the residue of the Bott class at each $U_{i}$ is well-defined as an element of $H_{c}^{2 q+1}\left(U_{i} ; \boldsymbol{C} / \boldsymbol{Z}\right)$ for each $i$. If we denote the residue by $\operatorname{res} B_{q}(\mathcal{F}, X, e)_{i}$, then $\sum_{i=1}^{r} \iota_{i *} \operatorname{res} B_{q}(\mathcal{F}, X, e)_{i}=B_{q}(\mathcal{F})$, where $\iota_{i *}$ denotes the mapping from $H_{c}^{2 q+1}\left(U_{i} ; C / Z\right)$ to $H^{2 q+1}(M ; \boldsymbol{C} / \boldsymbol{Z})$ defined as the extension by zero.

Proof. Let $\nabla_{1}^{X}$ and $\nabla_{2}^{X}$ be two basic $X$-connections, then $B_{q}\left(\nabla_{1}^{X}\right)-B_{q}\left(\nabla_{2}^{X}\right)$ is of the form $\sum D \omega_{k}$, where $\omega_{k}$ is given by 3) of Lemma 2.16. The Bott vanishing theorem for $\mathcal{F}_{X}$ shows that $\omega_{0}=0$ on $U$. If $k>1$, then $\omega_{k}=0$ on $U$ because $\Theta=0$ on $U$. Hence $B_{q}\left(\nabla_{1}^{X}, e\right)$ and $B_{q}\left(\nabla_{2}^{X}, e\right)$ determine the same compact cohomology class. Let now $e$ and $e^{\prime}$ be two trivializations of $-K_{\mathcal{F}}$ on $U$, then $B_{q}\left(\nabla, e^{\prime}\right)-B_{q}(\nabla, e)$ is of the form $\sum D \sigma_{i}+\sum D \rho_{k}$ as in 2) of Lemma 2.16. If we assume that $e$ and $e^{\prime}$ are homotopic on $U$, then the cochain $\nu$ must be equal to zero by continuity. Hence $\sigma_{i}$, $i>0$, and all $\rho_{k}$ 's are zero. The equation $\sigma_{0}=0$ holds again by the Bott vanishing theorem for $\mathcal{F}_{X}$. The rest of the claims are now clear.

REMARK 3.5. The residue is in fact an element of $\varliminf H^{2 q+1}\left(U_{X} ; \boldsymbol{C} / \boldsymbol{Z}\right)$, where $U_{X}$ runs through the open sets with the properties that $U_{X} \supset Z_{X}$ and that there is a trivialization $e_{X}$ on a neighborhood of $M \backslash U_{X}$. The partial order is defined by $\left(U_{X}, e_{X}\right)>\left(U_{X}^{\prime}, e_{X}^{\prime}\right)$ if $U_{X} \subset U_{X}^{\prime}$ and $e_{X}^{\prime}$ is the restriction of $e_{X}$.

The residue res $B_{q}(\mathcal{F}, X, e)$ is the $\bmod Z$-reduction of Heitsch's residue.

Proposition 3.6. Assume that there is a global trivialization e of $-K_{\mathcal{F}}$ and let $X$ be a $\Gamma$-vector field for $\mathcal{F}$, then $\operatorname{res} B_{q}(\mathcal{F}, X, e)=\operatorname{res}_{\hat{c}_{1} c_{1}^{q}}(\mathcal{F}, X, e) \bmod \boldsymbol{Z}$, where res $_{\hat{c}_{1} c_{1}^{q}}(\mathcal{F}, X, e)$ is Heitsch's residue defined in [18].

Proof. Let $\Psi_{0}$ be a smooth function with the following properties:

1) $0 \leq \Psi_{0} \leq 1$

2) $\left.\Psi_{0}\right|_{U_{X} \backslash U}=0$, and

3) $\left.\Psi_{0}\right|_{U \backslash U_{X}}=1$.

We set $\Psi_{1}=1-\Psi_{0}$. Let $\nabla_{0}$ and $\nabla_{1}$ be Bott connections on $U_{X}$ and $U$, respectively. Precisely speaking, it is necessary to consider refinements of the covering $\left\{U_{X}, U\right\}$ in the following arguments. But we omit it for simplify the exposition. 
Define a global connection $\nabla^{g}$ by $\nabla^{g}=\left(1-\Psi_{0}\right) \nabla_{0}+\Psi_{0} \nabla_{1}$. Note that if $\nabla=$ $\left\{\nabla_{0}, \nabla_{1}\right\}$ is a basic $X$-connection, then $\nabla^{g}$ is a global basic $X$-connection. Let $\widetilde{\zeta}^{g}$ be the connection form of $\nabla^{g}$ with respect to $e$, and set $\zeta^{g}=(-2 \pi \sqrt{-1})^{-1} \widetilde{\zeta}^{g}$, then Heitsch's residue is by definition the compact support cohomology class represented by $\zeta^{g} \wedge\left(d \zeta^{g}\right)^{q}$. Now by definition $\beta=\left\{\beta_{01}\right\}$, where $\beta_{01}=\zeta_{1}-\zeta_{0}$. Define a Cech de Rham $(0,1)$-cochain $(\Psi \beta)$ by $(\Psi \beta)=\left\{\Psi_{0} \beta_{01},-\Psi_{1} \beta_{01}\right\}$, then $D(\Psi \beta)=-\beta+d(\Psi \beta)$ and $\zeta^{g}=\zeta_{0}+\Psi_{0}\left(\zeta_{1}-\zeta_{0}\right)=\zeta_{1}-\Psi_{1}\left(\zeta_{1}-\zeta_{0}\right)$. Hence $\zeta^{g}=\zeta+(\Psi \beta)$, where $\zeta=\left\{\zeta_{0}, \zeta_{1}\right\}$, and $B_{q}(\nabla, e)=\zeta \cup(d \zeta+\beta)^{q}=\zeta^{g} \cup(d \zeta+\beta)^{q}$ because $(\Psi \beta) \cup(d \zeta+\beta)^{q}=0$ by degree reason in the normal direction. Set $\omega=\zeta^{g} \cup \Delta_{(\Psi \beta)}\left((d \zeta+d(\Psi \beta))^{q},(d \zeta+\beta)^{q}\right)$, then $d \zeta^{g} \cup \Delta_{(\Psi \beta)}\left((d \zeta+d(\Psi \beta))^{q},(d \zeta+\beta)^{q}\right)=0$ again by the degree reason. It follows that $D \omega=\zeta^{g} \cup\left(d \zeta^{g}\right)^{q}-B_{q}(\nabla, e)$. Since supp $\omega \subset U_{0} \cap U_{1}$, these cocycles define the same compact support cohomology class.

The following corollary originally due to Heitsch shows that the residue has a close relationship with the holonomy of leaves.

Corollary 3.7. Let $\mathcal{F}$ be the foliation of $S^{1} \times C^{q}$ induced by the vector field $T=$ $\frac{\partial}{\partial t}+\lambda_{1} z_{1} \frac{\partial}{\partial z_{1}}+\cdots+\lambda_{q} z_{q} \frac{\partial}{\partial z_{q}}$, where $t$ and $z=\left(z_{1}, \cdots, z_{q}\right)$ are the natural coordinates of $\boldsymbol{R}$ and $\boldsymbol{C}^{q}$, respectively, and $S^{1}$ is considered as $\boldsymbol{R} / \boldsymbol{Z}$. Let $X=\delta_{1} z_{1} \frac{\partial}{\partial z_{1}}+\cdots+$ $\delta_{q} z_{q} \frac{\partial}{\partial z_{q}}$ and $e=e^{2 \pi \sqrt{-1} k t} \frac{\partial}{\partial z_{1}} \wedge \cdots \wedge \frac{\partial}{\partial z_{q}}$, where $k \in Z$. Then

$$
\operatorname{res} B_{q}(\mathcal{F}, X, e)=\left(\frac{\lambda_{1}+\cdots+\lambda_{q}}{2 \pi \sqrt{-1}}-k\right) \frac{\left(\delta_{1}+\cdots+\delta_{q}\right)^{q}}{\delta_{1} \cdots \delta_{q}} d t \quad \bmod Z
$$

REMARK 3.8. Heitsch proved a more general formula that involves the Grothendieck residue (Theorem 3.1 of [18]), which is also valid in our case after mod- $\boldsymbol{Z}$ reduction.

4. Relation to Differential characters. There is another representation of the Bott class which helps us to study the properties of the Bott class as a Differential character. Let $e=\left\{e_{i}\right\}$ be a family of local trivializations of $-K_{\mathcal{F}}$ and let $\left\{\tilde{\alpha}_{i j}\right\}$ be a family of local functions such that $e_{i}=\widetilde{\alpha}_{i j} e_{j}$. Let $\nabla^{b}$ a globally well-defined Bott connection, and $\nabla^{u}$ be a globally well-defined unitary connection for some Hermitian metric of $-K_{\mathcal{F}}$. We denote by $\widetilde{\zeta}_{i}$ and $\widetilde{\eta}_{i}$ the connection form of $\nabla^{b}$ and $\nabla^{u}$ with respect to $e_{i}$, respectively. These connection forms satisfy $\delta \widetilde{\zeta}=\delta \widetilde{\eta}=-\frac{d \widetilde{\alpha}}{\widetilde{\alpha}}$. We normalize these cochains as in the previous sections: $\zeta=\frac{-1}{2 \pi \sqrt{-1}} \widetilde{\zeta}, \eta=\frac{-1}{2 \pi \sqrt{-1}} \tilde{\eta}$. Cochains which involve $\widetilde{\alpha}$ are also normalized in the same way as in Section 2. We now introduce the following differential forms: 
Definition 4.1. Set

$$
\begin{aligned}
& u_{1}\left(\nabla^{b}, \nabla^{u}\right)=\zeta-\eta=\frac{-1}{2 \pi \sqrt{-1}}(\tilde{\zeta}-\tilde{\eta}) \\
& \tilde{u}_{1}\left(\nabla^{b}, \nabla^{u}\right)=u_{1}\left(\nabla^{b}, \nabla^{u}\right)-\frac{u_{1}\left(\nabla^{b}, \nabla^{u}\right)}{=}=(\zeta-\bar{\zeta})-(\eta-\bar{\eta}) \\
& c_{1}\left(\nabla^{b}\right)=d \zeta=\frac{-1}{2 \pi \sqrt{-1}} d \zeta_{i} \\
& c_{1}\left(\nabla^{u}\right)=d \eta=\frac{-1}{2 \pi \sqrt{-1}} d \eta_{i}
\end{aligned}
$$

We define two $(2 q+1)$-forms by

$$
\begin{aligned}
& \widehat{B}_{q}\left(\nabla^{b}, \nabla^{u}\right) \\
= & u_{1}\left(\nabla^{b}, \nabla^{u}\right) \cup\left(c_{1}\left(\nabla^{b}\right)^{q}+c_{1}^{q-1}\left(\nabla^{b}\right) c_{1}\left(\nabla^{u}\right)+\cdots+c_{1}\left(\nabla^{u}\right)^{q}\right) \\
= & (\zeta-\eta) \cup\left((d \zeta)^{q}+(d \zeta)^{q-1} d \eta+\cdots+(d \eta)^{q}\right), \text { and } \\
& I B_{q}\left(\nabla^{b}, \nabla^{u}\right) \\
= & (2 \sqrt{-1})^{-1} \widetilde{u}_{1}\left(\nabla^{b}, \nabla^{u}\right) \cup\left(c_{1}\left(\nabla^{b}\right)^{q}+c_{1}\left(\nabla_{b}\right)^{q-1} \overline{c_{1}\left(\nabla^{b}\right)}+\cdots+\overline{c_{1}\left(\nabla^{b}\right)^{q}}\right) \\
= & (2 \sqrt{-1})^{-1}((\zeta-\bar{\zeta})-(\eta-\bar{\eta})) \cup\left((d \zeta)^{q}+(d \zeta)^{q-1} d \bar{\zeta}+\cdots+(d \bar{\zeta})^{q}\right) .
\end{aligned}
$$

Note that these differential forms are independent of the choice of local trivializations. Note also that Lemma 2.12 shows that $c_{1}\left(\nabla^{b}\right)$ represents the image of $c_{1}\left(-K_{\mathcal{F}}\right)$ in $H^{2}(M ; C)$. This is also the case for $c_{1}\left(\nabla^{u}\right)$ because $D((\eta-\log \alpha))=c_{1}\left(\nabla^{u}\right)+\delta \eta+$ $\frac{d \alpha}{\alpha}-\Theta=c_{1}\left(\nabla^{u}\right)-\Theta$.

The following fact is well-known.

TheOREM-Definition 4.2. IB $B_{q}\left(\nabla^{b}, \nabla^{u}\right)$ represents a class in $H^{2 q+1}(M ; \boldsymbol{R})$ independent of the choice of connections and of the choice of Hermitian metrics on $-K_{\mathcal{F}}$. This class is called the imaginary part of the Bott class and denoted by $I B_{q}(\mathcal{F})$.

REMARK 4.3. The class $I B_{q}(\mathcal{F})$ was first defined in [5]. In our previous papers, the class $\xi_{q}(\mathcal{F})=-2 I B_{q}(\mathcal{F})$ is used.

LEMMA 4.4. The differential form $\widehat{B}_{q}\left(\nabla^{b}, \nabla^{u}\right)$ determines a $C$-valued differential character which is independent of $\nabla^{b}$.

Proof. As $\widehat{B}_{q}\left(\nabla^{b}, \nabla^{u}\right)$ is a globally well-defined $(2 q+1)$-form, it determines a $C$-valued differential character. Let $\nabla^{b^{\prime}}$ be another Bott connection and denote by $\zeta^{\prime}$ the normalized local connection forms, then $\zeta^{\prime}-\zeta$ is a global 1-form of transverse type- $(1,0)$. We set for two 2 -forms $\omega$ and $\mu$

$$
(\omega, \mu)^{k}=\omega^{k}+\omega^{k-1} \cup \mu+\cdots+\mu^{k}
$$

Set $\rho=\left(\zeta^{\prime}-\eta\right) \cup\left(\zeta^{\prime}-\zeta\right) \cup\left(\left(d \zeta^{\prime}, d \zeta\right)^{q-1}+\left(d \zeta^{\prime}, d \zeta\right)^{q-2} \cup d \eta+\cdots+(d \eta)^{q-1}\right)$, then 
noticing that $\left(\zeta^{\prime}-\eta\right)$ and $\left(\zeta^{\prime}-\zeta\right)$ are globally well-defined,

$$
\begin{aligned}
& D \rho \\
= & -\left(\zeta^{\prime}-\eta\right) \cup\left(\left(d \zeta^{\prime}\right)^{q}+\left(d \zeta^{\prime}\right)^{q-1} \cup d \eta+\cdots+(d \eta)^{q}\right) \\
& +\left(\zeta^{\prime}-\eta\right) \cup\left((d \zeta)^{q}+(d \zeta)^{q-1} \cup d \eta+\cdots+(d \eta)^{q}\right) \\
& +\left(\zeta^{\prime}-\zeta\right) \cup\left(d \zeta^{\prime}-d \eta\right) \cup\left(\left(d \zeta^{\prime}, d \zeta\right)^{q-1}+\left(d \zeta^{\prime}, d \zeta\right)^{q-2} \cup d \eta+\cdots+(d \eta)^{q-1}\right) .
\end{aligned}
$$

Hence

$$
\begin{aligned}
& \widehat{B}_{q}\left(\nabla^{b^{\prime}}, \nabla^{u}\right)-\widehat{B}_{q}\left(\nabla^{b}, \nabla^{u}\right)+D \rho \\
= & \left(\zeta^{\prime}-\eta\right) \cup\left(\left(d \zeta^{\prime}\right)^{q}+\left(d \zeta^{\prime}\right)^{q-1} \cup d \eta+\cdots+(d \eta)^{q}\right) \\
& -(\zeta-\eta) \cup\left((d \zeta)^{q}+(d \zeta)^{q-1} \cup d \eta+\cdots+(d \eta)^{q}\right) \\
& +D \rho \\
= & \left(\zeta^{\prime}-\eta\right) \cup\left((d \zeta)^{q}+(d \zeta)^{q-1} \cup d \eta+\cdots+(d \eta)^{q}\right) \\
& -(\zeta-\eta) \cup\left((d \zeta)^{q}+(d \zeta)^{q-1} \cup d \eta+\cdots+(d \eta)^{q}\right) \\
& +\left(\zeta^{\prime}-\zeta\right) \cup\left(d \zeta^{\prime}-d \eta\right) \cup\left(\left(d \zeta^{\prime}, d \zeta\right)^{q-1}+\left(d \zeta^{\prime}, d \zeta\right)^{q-2} \cup d \eta+\cdots+(d \eta)^{q-1}\right) .
\end{aligned}
$$

On the other hand,

$$
\begin{aligned}
& \left(d \zeta^{\prime}-d \eta\right) \cup\left(\left(d \zeta^{\prime}, d \zeta\right)^{q-1}+\left(d \zeta^{\prime}, d \zeta\right)^{q-2} \cup d \eta+\cdots+(d \eta)^{q-1}\right) \\
= & \left(d \zeta^{\prime}\right)^{q}-(d \eta)^{q}+\left(\left(d \zeta^{\prime}\right)^{q-1}-(d \eta)^{q-1}\right) \cup d \zeta+\cdots+\left(d \zeta^{\prime}-d \eta\right) \cup(d \zeta)^{q-1}
\end{aligned}
$$

Thus

$$
\begin{aligned}
& \widehat{B}_{q}\left(\nabla^{b^{\prime}}, \nabla^{u}\right)-\widehat{B}_{q}\left(\nabla^{b}, \nabla^{u}\right)+D \rho \\
= & \left(\zeta^{\prime}-\zeta\right) \cup\left(\left(d \zeta^{\prime}\right)^{q}+\left(d \zeta^{\prime}\right)^{q-1} \cup d \zeta+\cdots+(d \zeta)^{q}\right),
\end{aligned}
$$

which is equal to zero because of the transversal degree. As exact forms are zero as differential characters, we are done.

We need another differential character.

Definition 4.5. Let $T_{q}\left(\nabla^{u}, e\right)$ be the Čech-de Rham cochain given by the formula

$$
\begin{aligned}
& T_{q}\left(\nabla^{u}, e\right) \\
= & (\eta-\log \alpha) \cup c_{1}\left(\nabla^{u}\right)^{q} \\
& +\Theta \cup(\eta-\log \alpha) \cup c_{1}\left(\nabla^{u}\right)^{q-1} \\
& +\cdots \\
& +\Theta^{q} \cup(\eta-\log \alpha) .
\end{aligned}
$$

REMARK 4.6. Suppose that $\nabla^{b}$ happens to be equal to $\nabla^{u}$, then $\widehat{B}_{q}\left(\nabla^{b}, \nabla^{u}\right)=0$ and $T_{q}\left(\nabla^{u}, e\right)=T_{q}\left(\nabla^{b}\right)$. This is indeed the case if $\mathcal{F}$ is transversely Hermitian.

LemMa 4.7. $T_{q}\left(\nabla^{u}, e\right)$ determines an $\boldsymbol{R} / \boldsymbol{Z}$-valued differential character via integration of Lehmann. As a differential character, $T_{q}\left(\nabla^{u}, e\right)$ is independent of the choice of $e$. 
Proof. First, $T_{q}\left(\nabla^{u}, e\right)$ determines a $C / Z$-valued differential character because $D T_{q}\left(\nabla^{u}, e\right)=-c_{1}\left(\nabla^{u}\right)^{q+1}+\Theta^{q+1}$. Let $e$ and $e^{\prime}$ be two families of local trivializations of $-K_{\mathcal{F}}$, then we may assume that $e_{i}^{\prime}=g_{i} e_{i}$ for some non-zero function $g_{i}$. We define $\widetilde{\alpha}^{\prime}=\left\{\widetilde{\alpha}_{i j}^{\prime}\right\}$ by requiring $e_{i}^{\prime}=\widetilde{\alpha}_{i j}^{\prime} e_{j}^{\prime}$, then $\widetilde{\alpha}_{i j}^{\prime}=g_{i} \widetilde{\alpha}_{i j} g_{j}^{-1}$. Hence there is a collection $\nu=\left\{\nu_{i j}\right\}$ of integers such that $\log \alpha^{\prime}-\log \alpha=-(-2 \pi \sqrt{-1})^{-1} \delta \log g+\nu$. By following a similar argument as in the proof of Lemma $2.16,2)$, one can show that $T_{q}\left(\nabla^{u}, e\right)$ and $T_{q}\left(\nabla^{u}, e^{\prime}\right)$ determine the same differential character. Now we choose $e$ as a collection of local normal basis, then $\eta-\bar{\eta}=0, \log \alpha-\overline{\log \alpha}=0$ and $c_{1}\left(\nabla^{u}\right)=\overline{c_{1}\left(\nabla^{u}\right)}$. Hence $T_{q}\left(\nabla^{u}, e\right)$ is $\boldsymbol{R} / \boldsymbol{Z}$-valued.

Definition 4.8. Let $\nabla^{b}$ be a Bott connection, $\nabla^{u}$ be a unitary connection for some Hermitian metric on $-K_{\mathcal{F}}$ and $e$ be a trivialization. We denote by $\widehat{B}_{q}\left(\mathcal{F}, \nabla^{u}\right)$ and $T_{q}\left(\nabla^{u}\right)$ the differential character determined by $\widehat{B}_{q}\left(\nabla^{b}, \nabla^{u}\right)$ and by $T_{q}\left(\nabla^{u}, e\right)$, respectively.

REMARK 4.9. Note that $T_{q}\left(\nabla^{u}\right)$ is determined only by $-K_{\mathcal{F}}$ and $\nabla^{u}$, and is independent of the foliation $\mathcal{F}$ itself.

These differential characters and the Bott class are related as follows:

Proposition 4.10. $\widehat{B}_{q}\left(\mathcal{F}, \nabla^{u}\right)+T_{q}\left(\nabla^{u}\right)$ is in fact an element of $H^{2 q+1}(M ; C / Z)$ and coincides with the Bott class.

Proof. This will follow by checking that $\widehat{B}_{q}\left(\mathcal{F}, \nabla^{u}\right)+T_{q}\left(\nabla^{u}\right)$ satisfies the characterization of the Bott class in Definition 2.8. But we give a direct proof for later use. First,

$$
\begin{aligned}
& \widehat{B}_{q}\left(\nabla^{b}, \nabla^{u}\right)+T_{q}\left(\nabla^{u}, e\right) \\
= & (\zeta-\eta) \cup\left((d \zeta)^{q}+(d \zeta)^{q-1} d \eta+\cdots+(d \eta)^{q}\right) \\
& +(\eta-\log \alpha) \cup(d \eta)^{q} \\
& +\Theta \cup(\eta-\log \alpha) \cup(d \eta)^{q-1} \\
& +\cdots \\
& +\cdots \\
& +\Theta^{q} \cup(\eta-\log \alpha) .
\end{aligned}
$$

Set

$$
\begin{aligned}
\omega_{k}= & \Theta^{k} \cup \zeta \cup \eta \cup\left((d \zeta)^{q-k-1}+(d \zeta)^{q-k-2} d \eta+\cdots+(d \eta)^{q-k-1}\right) \\
& +\Theta^{k} \cup \log \alpha \cup(\zeta-\eta) \cup\left((d \zeta)^{q-k-1}+(d \zeta)^{q-k-2} d \eta+\cdots+(d \eta)^{q-k-1}\right),
\end{aligned}
$$

then

$$
\begin{aligned}
D \omega_{k}= & \Theta^{k} \cup(\eta \cup d \zeta-\zeta \cup d \eta) \cup\left((d \zeta)^{q-k-1}+(d \zeta)^{q-k-2} d \eta+\cdots+(d \eta)^{q-k-1}\right) \\
& +\Theta^{k} \cup \frac{d \alpha}{\alpha} \cup(\zeta-\eta) \cup\left((d \zeta)^{q-k-1}+(d \zeta)^{q-k-2} d \eta+\cdots+(d \eta)^{q-k-1}\right) \\
& -\Theta^{k} \cup \frac{d \alpha}{\alpha} \cup(\zeta-\eta) \cup\left((d \zeta)^{q-k-1}+(d \zeta)^{q-k-2} d \eta+\cdots+(d \eta)^{q-k-1}\right) \\
& +\Theta^{k+1} \cup(\zeta-\eta) \cup\left((d \zeta)^{q-k-1}+(d \zeta)^{q-k-2} d \eta+\cdots+(d \eta)^{q-k-1}\right) \\
& -\Theta^{k} \cup \log \alpha \cup\left((d \zeta)^{q-k}-(d \eta)^{q-k}\right) .
\end{aligned}
$$


It is easy to verify that

$$
\widehat{B}_{q}\left(\nabla^{b}, \nabla^{u}\right)+T_{q}\left(\nabla^{u}, e\right)+D \omega_{0}+\cdots+D \omega_{q-1}=B_{q}\left(\nabla^{b}, e\right) .
$$

5. Codimension-one case. In [2], we defined another type of residue for $I B_{q}(\mathcal{F})$. The definition is based on the fact that we may assume $\nabla^{b}=\nabla^{u}$ if $\mathcal{F}$ is transversely Hermitian, that is, $\mathcal{F}$ admits a holonomy invariant Hermitian metric. This residue is known to coincide with Heitsch's one if the complex codimension is equal to one (Theorem 5.9 of [2]). As in Section 3, we assume that there is a trivialization of $-K_{\mathcal{F}}$ on an open set. Then a residue which is the same type as in [2] can be defined as follows:

DeFinition 5.1. Suppose that there is an open set $U$ on which $\mathcal{F}$ is transversely Hermitian and $-K_{\mathcal{F}}$ admits a trivialization $e_{U}$. Let $V$ be a neighborhood of $M \backslash U$, and $U^{\prime}$ be a neighborhood of $M \backslash V$. Let $\nabla^{b}$ and $\nabla^{u}$ be a Bott connection and a unitary connection such that $\nabla^{b}=\nabla^{u}$ on $U^{\prime}$, and $e$ be a family of trivialization of $-K_{\mathcal{F}}$ which coincides with $e_{U}$ on $U^{\prime}$. We set

$$
\operatorname{res}_{V}^{*} B_{q}(\mathcal{F}, e)=\left[\widehat{B}_{q}\left(\nabla^{b}, \nabla^{u}\right)+T_{q}\left(\nabla^{u}, e\right)\right] \in H_{c}^{2 q+1}(V ; C / Z) .
$$

One can show that res ${ }^{*} B_{q}(\mathcal{F}, e)$ is independent of the choice of connections and transverse Hermite structure by considering families of connections and metrics (cf. [2]). This argument is standard and we omit it.

Note that we can find a holonomy invariant trivialization of $-K_{\mathcal{F}}$ on $U$. Since the complex codimension of $\mathcal{F}$ is equal to one, this trivialization can be seen as a $\Gamma$-vector field, which we denote by $X$.

Then, in fact, we have the following:

THEOREM 5.2. If the complex codimension of $\mathcal{F}$ is equal to one, then the two residues are the same, namely,

$$
\operatorname{res}_{V}^{*} B_{q}(\mathcal{F}, e)=\operatorname{res} B_{q}(\mathcal{F}, X, e) \in H_{c}^{2 q+1}(V ; C / Z) .
$$

In particular, the residue depends on $\left.\mathcal{F}\right|_{U_{X}}$, where $U_{X}$ is as in the previous section, but not on the particular choice of $X$.

Proof. Choose a basic- $X$ connection $\nabla^{X}$ as a Bott connection $\nabla^{b}$, where we assume that $\nabla^{X}$ is globally defined. Then $\nabla^{X}=\nabla^{b}$ can be seen as a unitary connection for an appropriate Hermitian metric on $-K_{\mathcal{F}}$ defined by using $X$ (see the proof of Theorem 5.9 of [2]). Noticing that cochains $\omega_{k}$ in the proof of Proposition 4.10 is equal to zero if $\nabla^{b}=\nabla^{u}$, we see that the two cocycles $\widehat{B}\left(\nabla^{X}, \nabla^{X}\right)+T\left(\nabla^{X}, e\right)$ and $B_{q}\left(\nabla^{X}, e\right)$ determine the same element of $H_{c}^{2 q+1}(V ; C / Z)$. The last statement follows from the fact that $\operatorname{res}_{V}^{*} B_{q}(\mathcal{F}, e)$ is independent of $X$.

There is a structure theorem for transversely holomorphic foliations of complex codimension one due to Ghys, Gomez-Mont and Saludes. We quote it in a reduced form:

THEOREM 5.3 [16]. Let $\mathcal{F}$ be a transversely holomorphic foliation of a closed manifold $M$, of complex codimension one. Then there is a unique decomposition of $M$ into three parts $M=F_{0} \cup F_{L} \cup J$ such that $\mathcal{F}$ restricted to $F_{0} \cup F_{L}$ is transversely Hermitian and $\mathcal{F}$ restricted to $F_{L}$ is transversely Lie, where $F_{0}$ is an open set called 
the wandering Fatou component, $F_{L}$ is the union of the rest of the Fatou components (and thus an open set), and $J$ is a closed set called the Julia component.

Since transversely Lie foliations are naturally transversely Hermitian and they admit holonomy invariant trivializations of $-K_{\mathcal{F}}$, we have the following weak version of Duminy's theorem [10]:

COROLlary 5.4. The Bott class is determined by the germ of the foliation at $F_{0} \cup J$. Particularly if $F_{0} \cup J$ is empty, then the Bott class is trivial.

6. Futaki Invariant. Let $M$ be a compact complex manifold of complex dimension $q$. The Futaki invariant is a group homomorphism from $\operatorname{Aut}(M)$ to $\boldsymbol{C} / \boldsymbol{Z}$, where $\operatorname{Aut}(M)$ denotes the group of biholomorphic diffeomorphisms of $M$. It is defined as follows. First, let $\mathcal{F}_{\sigma}$ be the suspension of $\sigma$ as in Example 2.4.

DeFinition $6.1[14]$. Set

$$
F(\sigma)=\left\langle B_{q}\left(\mathcal{F}_{\sigma}\right),\left[M_{\sigma}\right]\right\rangle,
$$

where $B_{q}\left(\mathcal{F}_{\sigma}\right)$ is the Bott class of $\mathcal{F}_{\sigma}$ and $\left[M_{\sigma}\right]$ is the fundamental cycle of $M_{\sigma}$.

Recall now that the Bott class is decomposed as $\widehat{B}_{q}+T_{q}$ as a differential character. This decomposition is quite natural when Futaki invariant is discussed. As we are concerned with mapping tori such as $M_{\sigma}$, there is a natural choice of connections. Namely, under the notations in [14], we may choose Bott and unitary connections as $\nabla^{b}=h_{t}^{-1} \partial h_{t}$ and $\nabla^{u}=h_{t}^{-1} \partial h_{t}+\frac{1}{2} h_{t}^{-1} \partial_{t} h_{t}$ (which are the same choice as in [14]). It follows that $u_{1}\left(\nabla^{b}, \nabla^{u}\right)=(2 c)^{-1} \partial_{t} \log h_{t}$ and that $c_{1}\left(\nabla^{b}\right)=c_{1}\left(\nabla^{u}\right)=$ $c^{-1} \bar{\partial} \partial \log h_{t} \bmod d t$, where $c=-2 \pi \sqrt{-1}$. In this case, $\widehat{B}_{q}\left(\nabla^{b}, \nabla^{u}\right)$ is $\sqrt{-1} R$-valued because $\widehat{B}_{q}\left(\nabla^{b}, \nabla^{u}\right)=\frac{1}{2} c^{-q-1}\left(\partial_{t} \log h_{t}\right) \wedge\left(\bar{\partial} \partial \log h_{t}\right)^{q}$. On the other hand, $T_{q}\left(\nabla^{u}\right)$ is $R / Z$-valued by Lemma 4.7. Therefore, the decomposition $B_{q}=\widehat{B}_{q}+T_{q}$ gives the decomposing of $B_{q}$ into real and imaginary parts.

In view of this fact, we introduce the following

DEFINITION 6.2. A transverse unitary connection $\nabla^{u}$ on $-K_{\mathcal{F}}$ is said to be adapted if $\widehat{B}_{q}\left(\nabla^{b}, \nabla^{u}\right)$ is $\sqrt{-1} R$-valued.

We have the following

TheOREM 6.3. Let $M, \sigma, M_{\sigma}$ and $\mathcal{F}_{\sigma}$ as above. Let $\nabla^{u}$ be any transverse unitary connection for some Hermitian metric on $-K_{\mathcal{F}}$. Suppose that $\nabla^{u}$ is adapted, then $\widehat{B}_{q}\left(\mathcal{F}_{\sigma}, \nabla^{u}\right)$ and $T_{q}\left(\nabla^{u}\right)$ respectively determine differential characters independent of the choice of metric and $\nabla^{u}$. In fact, $\widehat{B}_{q}\left(\mathcal{F}_{\sigma}, \nabla^{u}\right)=\sqrt{-1} \operatorname{Im} B_{q}\left(\mathcal{F}_{\sigma}\right)$ and $T_{q}\left(\nabla^{u}\right)=$ $\operatorname{Re} B_{q}\left(\mathcal{F}_{\sigma}\right)$.

Proof. It follows from Proposition 4.10 that $\widehat{B}_{q}\left(\mathcal{F}_{\sigma}, \nabla^{u}\right)+T_{q}\left(\nabla^{u}\right)$ is the same as $B_{q}\left(\mathcal{F}_{\sigma}\right)$ as $\boldsymbol{C} / \boldsymbol{Z}$-valued differential characters. As $T_{q}\left(\nabla^{u}\right)$ is always a $\boldsymbol{R} / \boldsymbol{Z}$-valued differential character by Lemma 4.7 , the assertion is just a consequence of Definition 6.2 .

An integration formula for the imaginary part of $F$ is well-known [14]. It is still difficult to deduce an effective integration formula for the real part of $F$, but we have the following formula: 
Corollary 6.4. If $\nabla^{u}$ is adapted, then

$$
F(\sigma)=\int_{M_{\sigma}} B_{q}\left(\mathcal{F}_{\sigma}\right)=\int_{M_{\sigma}} T_{q}\left(\nabla^{u}\right)+\sqrt{-1} \int_{M_{\sigma}} I B_{q}\left(\mathcal{F}_{\sigma}\right),
$$

where the integration of $B_{q}\left(\mathcal{F}_{\sigma}\right)$ and $T_{q}\left(\nabla^{u}\right)$ is in the sense of Lehmann. The second equality gives the decomposition of $F$ into the real and imaginary parts.

REMARK 6.5. One can verify that the formula given in [14] can be obtained from the integration $\int_{M_{\sigma}} I B_{q}\left(\mathcal{F}_{\sigma}\right)$. An advantage of this expression is that one need not prepare globally well-defined connections, which will often involve a partition of unity. Note that $I B_{q}\left(\mathcal{F}_{\sigma}\right)$ depends on $\mathcal{F}_{\sigma}$ but $T_{q}$ depends only on $-K_{\mathcal{F}_{\sigma}}$. More precisely, we need the Bott connection in order to define $I B_{q}\left(\mathcal{F}_{\sigma}\right)$. As the Bott connection can be seen as the differentiation along the leaves, one can say that $I B_{q}\left(\mathcal{F}_{\sigma}\right)$ reflects dynamical properties of $\sigma$. On the other hand, $T_{q}\left(\nabla^{u}\right)$ is less dependent on Bott connection and thus on the dynamical properties of $\sigma$.

Corollary 6.4 has some other corollaries, which might be well-known for specialists. The first one is a consequence of the second expression.

Corollary 6.6. Suppose that $\sigma \in \operatorname{Aut}(M)$ preserves a Hermitian metric on $M$, then $F(\sigma) \in \boldsymbol{R} / \boldsymbol{Z}$. This is the case if $M$ is Kähler and $\sigma$ is a Kähler automorphism.

Proof. Note that in this case we may assume that $\nabla^{b}=\nabla^{u}$, because the foliation $\mathcal{F}_{\sigma}$ is transversely Hermitian. It follows that $I B_{q}\left(\mathcal{F}_{\sigma}\right)=0$.

The first expression has the following

Corollary 6.7. Let $\sigma \in \operatorname{Aut}(M)$. Suppose that the canonical bundle $K_{M}$ is trivial as a smooth bundle and that there is a non-vanishing holomorphic vector field invariant under the action of $\sigma$, then $F(\sigma)=0$.

Proof. As $K_{M}$ is trivial the terms concerning $\log \alpha$ and $\Theta$ vanish, because $K_{\mathcal{F}}$ is the pull-back of $K_{M}$. On the other hand, by the invariance, the vector field $X$ induces a $\Gamma$-vector field $X_{\sigma}$ on $M_{\sigma}$. Hence the rest of the cocycle given in Definition 2.13 also vanishes.

We do not know what this corollary suggests in the non-Kähler category. An example of a family of non-Kähler manifolds with vanishing $F$ is given in Example 7.6 .

We have some remarks

REMARK 6.8.

1) The first expression in Corollary 6.4 means that the Futaki invariant can be calculated by using local objects such as local connections, etc. In particular, one can consider the following situation: let $\sigma \in \operatorname{Aut}(M)$ and suppose that $M$ contains a $\sigma$-invariant open set $U$ on which $\sigma$ is of the form $\left.\sigma\right|_{U}=\exp X$ for some vector field $X$ on $U$. In such a case, let $V$ be a neighborhood of $M \backslash U$ and take a refinement of the covering $\{U, V\}$. As $B_{q}\left(\mathcal{F}_{\sigma}\right)$ can be calculated in terms of local objects, we can calculate $\left.B_{q}\left(\mathcal{F}_{\sigma}\right)\right|_{U}$ by using the vector field $X$. This fact will be used in calculations of examples.

2) As $H^{2 q+2}\left(M_{\sigma} ; Z\right)=\{0\}$ by dimension reason, there is a lift $\widetilde{F}(\sigma)$ valued in $C$ obtained by considering a lift of $B\left(\mathcal{F}_{\sigma}\right)$ in $H^{2 q+1}(M ; C)$. Indeed, Tsuboi showed 
under a certain assumption that a formula of the form $F(\sigma)=(1 / 2) \eta \bmod Z$ holds (Theorem 2.5 in [24]). Thus the $\eta$-invariants can be considered as the lift in this case.

7. Examples. We begin by checking Corollary 3.7 when the complex codimension is equal to one or two.

EXAMPLE 7.1. Let $\mathcal{F}$ be the foliation of $S^{1} \times C$ induced by the vector field $T_{\lambda}$ defined by

$$
T_{\lambda}=\frac{\partial}{\partial t}+\lambda z \frac{\partial}{\partial z}
$$

where $S^{1}$ is considered as $\boldsymbol{R} / \boldsymbol{Z}$, and $t, z$ are the natural coordinates of $\boldsymbol{R}, \boldsymbol{C}$, respectively. We denote by $D$ the closed unit disc in $\boldsymbol{C}$.

Let $X=\delta z \frac{\partial}{\partial z}$, then $X$ is a $\Gamma$-vector field for $\mathcal{F}$. Set $U_{X}=S^{1} \times 2 \epsilon D$ and $U=S^{1} \times(C \backslash \epsilon D)$, and adopt $e=e^{2 \pi \sqrt{-1} k t} \frac{\partial}{\partial z}$ as a trivialization of $-K_{\mathcal{F}}$, where $k \in Z$. A basic $X$-connection is given by $\nabla_{0}=-(\lambda-2 \pi \sqrt{-1} k) d t$ and $\nabla_{1}=-\frac{d z}{z}$. It follows that $\log \alpha=0$ and $\beta=\left(c^{-1} \lambda+k\right) d t-c^{-1} \frac{d z}{z}$, where $c=-2 \pi \sqrt{-1}$. By definition $B_{1}(\nabla, e)=-\left(c^{-1} \lambda+k\right) d t \cup\left(\left(c^{-1} \lambda+k\right) d t-c^{-1} \frac{d z}{z}\right)$, which is the differential form $-c^{-1}\left(c^{-1} \lambda+k\right) d t \wedge \frac{d z}{z}$ defined on $U_{X} \cap U$. It is easy to see that the integration of $B_{1}(\nabla, e)$ along the fiber of $S^{1} \times U_{X} \rightarrow S^{1}$ is given by $\oint-c^{-1}\left(c^{-1} \lambda+k\right) d t \wedge \frac{d z}{z}$ mod $Z$, where the integral path (circle) is chosen in $U_{X} \cap U$. This is clearly equal to $\frac{1}{2 \pi \sqrt{-1}} \lambda[d t] \bmod Z$, and is independent of $\delta$. It is also independent of $k$ in this case.

EXAmple 7.2. Consider the foliation of $S^{1} \times C^{2}$ defined by the vector field

$$
T=\frac{\partial}{\partial t}+\lambda_{1} z_{1} \frac{\partial}{\partial z_{1}}+\lambda_{2} z_{2} \frac{\partial}{\partial z_{2}}
$$

Let $X=\delta_{1} z_{1} \frac{\partial}{\partial z_{1}}+\delta_{2} z_{2} \frac{\partial}{\partial z_{2}}$, and let $U_{X}$ and $U$ as in Example 7.1 but $D$ is now the unit ball in $C^{2}$. We adopt $e=e^{2 \pi \sqrt{-1} k t} \frac{\partial}{\partial z_{1}} \wedge \frac{\partial}{\partial z_{2}}$ as a global trivialization of $-K_{\mathcal{F}}$. A basic $X$-connection is given by $\nabla_{0}=\left(2 \pi \sqrt{-1} k-\left(\lambda_{1}+\lambda_{2}\right)\right) d t$ and $\nabla_{1}=$ $\left(2 \pi \sqrt{-1} k-\left(\lambda_{1}+\lambda_{2}\right)\right) d t-\left(\delta_{1}+\delta_{2}\right) \omega$, where

$$
\omega=\frac{1}{\left|z_{1}\right|^{2}+\left|z_{2}\right|^{2}}\left(\frac{1}{\delta_{1}} \bar{z}_{1} d z_{1}+\frac{1}{\delta_{2}} \bar{z}_{2} d z_{2}-\left(\frac{\lambda_{1}}{\delta_{1}}\left|z_{1}\right|^{2}+\frac{\lambda_{2}}{\delta_{2}}\left|z_{2}\right|^{2}\right) d t\right) .
$$

It follows that $\beta=-\left(\delta_{1}+\delta_{2}\right) \omega$ and that

$$
\begin{aligned}
& B_{2}(\nabla, e) \\
= & c^{-3}\left(2 \pi \sqrt{-1} k-\left(\lambda_{1}+\lambda_{2}\right)\right) d t \cup\left(-\left(\delta_{1}+\delta_{2}\right) \omega\right) \cup\left(-\left(\delta_{1}+\delta_{2}\right) d \omega\right) \\
= & c^{-3}\left(2 \pi \sqrt{-1} k-\left(\lambda_{1}+\lambda_{2}\right)\right)\left(\delta_{1}+\delta_{2}\right)^{2} d t \cup \omega \cup d \omega \\
= & -c^{-3}\left(2 \pi \sqrt{-1} k-\left(\lambda_{1}+\lambda_{2}\right)\right)\left(\delta_{1}+\delta_{2}\right)^{2} d t \wedge \omega \wedge d \omega,
\end{aligned}
$$


which is a 4-form defined on $U_{X} \cap U$, where $c=2 \pi \sqrt{-1}$. After some calculations, one sees that

$$
\begin{aligned}
& \left.\omega \wedge d \omega\right|_{\left\{\left|z_{1}\right|^{2}+\left|z_{2}\right|^{2}=\epsilon^{2}\right\}} \bmod d t \\
= & \frac{1}{\epsilon^{4} \delta_{1} \delta_{2}}\left(\bar{z}_{1} d z_{1} \wedge d \bar{z}_{2} \wedge d z_{2}+\bar{z}_{2} d z_{2} \wedge d \bar{z}_{1} \wedge d z_{1}\right),
\end{aligned}
$$

and its integration is $\frac{c^{2}}{\delta_{1} \delta_{2}}$. Therefore, the integration of res $B_{2}(\mathcal{F}, X)$ along the fiber of $S^{1} \times D \rightarrow S^{1}$ is given by

$$
\left(\frac{1}{2 \pi \sqrt{-1}}\left(\lambda_{1}+\lambda_{2}\right)-k\right) \frac{\left(\delta_{1}+\delta_{2}\right)^{2}}{\delta_{1} \delta_{2}} d t
$$

Note that it does depend on $X$ and $k$.

The Futaki invariant is calculated as follows:

EXAMPLE 7.3 Let $\left\{V_{0}, V_{1}, V_{2}\right\}$ be the usual open covering of $C P^{2}$, that is, let $\left[t_{0}: t_{1}: t_{2}\right]$ be the homogenous coordinate and set $V_{i}=\left\{t_{i} \neq 0\right\}$. Let $\left(z_{1}, z_{2}\right),\left(u_{1}, u_{2}\right)$ and $\left(x_{1}, x_{2}\right)$ denote the coordinate of $V_{0}, V_{1}$ and $V_{2}$, respectively. We blow up $C P^{2}$ at the origin of $V_{0}$, namely, let $\widetilde{\boldsymbol{C}}^{2}=\left\{\left(\left(z_{1}, z_{2}\right),\left[t_{1}: t_{2}\right]\right) \mid t_{1} z_{2}-t_{2} z_{1}=0\right\}$ be $\boldsymbol{C}^{2}$ blown up at the origin and replace $V_{0}$ by $\widetilde{\boldsymbol{C}}^{2}$. Denote by $\widetilde{\boldsymbol{C} P^{2}}$ the resulting manifold and by $p: \widetilde{C P^{2}} \rightarrow C P^{2}$ the natural projection.

Consider now the vector field $X_{\lambda}$ on $C^{2}$ defined by

$$
X_{\lambda}=\lambda_{1} z_{1} \frac{\partial}{\partial z_{1}}+\lambda_{2} z_{2} \frac{\partial}{\partial z_{2}}
$$

which is lifted to $\widetilde{\boldsymbol{C} P^{2}}$ as follows. Let $U_{i}=\left\{\left(\left(z_{1}, z_{2}\right),\left[t_{1}: t_{2}\right]\right) \in \widetilde{\boldsymbol{C}}^{2} \mid t_{i} \neq 0\right\}$, then $U_{i} \cong C^{2}$. Indeed, the mapping $\varphi_{1}: U_{1} \rightarrow C^{2}$ defined by $\varphi\left(\left(\left(z_{1}, z_{2}\right),\left[t_{1}: t_{2}\right]\right)\right)=$ $\left(z_{1}, t_{2} / t_{1}\right)$ and $\varphi_{2}: U_{2} \rightarrow \boldsymbol{C}^{2}$ defined by $\varphi_{2}\left(\left(\left(z_{1}, z_{2}\right),\left[t_{1}: t_{2}\right]\right)\right)=\left(z_{2}, t_{1} / t_{2}\right)$ give the identifications. Let $\left(a_{1}, a_{2}\right)$ and $\left(b_{1}, b_{2}\right)$ be the coordinates of the targets of $\varphi_{1}$ and $\varphi_{2}$, respectively. Denote by $\iota$ the inclusion of $C^{2} \backslash\{0\}$ to $\tilde{C}^{2}$, and by $\iota_{i}$ the composition $\varphi_{i} \circ \iota$, then

$$
\begin{aligned}
\iota_{1 *} X_{\lambda} & =\lambda_{1} a_{1} \frac{\partial}{\partial a_{1}}+\left(\lambda_{2}-\lambda_{1}\right) a_{2} \frac{\partial}{\partial a_{2}} \text { and } \\
\iota_{2 *} X_{\lambda} & =\lambda_{2} b_{1} \frac{\partial}{\partial b_{1}}+\left(\lambda_{1}-\lambda_{2}\right) b_{2} \frac{\partial}{\partial b_{2}} .
\end{aligned}
$$

Remark that $\iota_{2}^{-1} \circ \iota_{1}\left(a_{1}, a_{2}\right)=\left(a_{1} a_{2}, 1 / a_{2}\right)$ and $\iota_{1}^{-1} \circ \iota_{2}\left(b_{1}, b_{2}\right)=\left(b_{1} b_{2}, 1 / b_{2}\right)$. Remark also that

$$
\frac{\partial}{\partial a_{1}}=\frac{1}{b_{2}} \frac{\partial}{\partial b_{1}} \text { and } \frac{\partial}{\partial a_{2}}=b_{1} b_{2} \frac{\partial}{\partial b_{1}}-b_{2}^{2} \frac{\partial}{\partial b_{2}}
$$

where we omitted to write $\left(\iota_{2}^{-1} \circ \iota_{1}\right)_{*}$.

On $V_{1}$ and $V_{2}$, the vector field $X_{\lambda}$ is given by

$$
\begin{aligned}
& -\lambda_{1} u_{1} \frac{\partial}{\partial u_{1}}+\left(\lambda_{2}-\lambda_{1}\right) u_{2} \frac{\partial}{\partial u_{2}} \text { on } V_{1} \text {, and } \\
& -\lambda_{2} x_{1} \frac{\partial}{\partial x_{1}}+\left(\lambda_{1}-\lambda_{2}\right) x_{2} \frac{\partial}{\partial x_{2}} \text { on } V_{2} .
\end{aligned}
$$


Let $\sigma$ be the automorphism of $\widetilde{\boldsymbol{C} P^{2}}$ induced by $X_{\lambda}$ and denote by $\mathcal{F}_{\sigma}$ the corresponding foliation of $\widetilde{C P^{2}}{ }_{\sigma}$, then $\mathcal{F}_{\sigma}$ restricted to $S^{1} \times \widetilde{\boldsymbol{C}}^{2}$ is spanned by the vector field $T=\frac{\partial}{\partial t}+X_{\lambda}$. Set $Y=\delta_{1} z_{1} \frac{\partial}{\partial z_{1}}+\delta_{2} z_{2} \frac{\partial}{\partial z_{2}}$, then $Y$ is a $\Gamma$-vector field for $\mathcal{F}_{\sigma}$. Finally, let $e_{0}=\frac{\partial}{\partial z_{1}} \wedge \frac{\partial}{\partial z_{2}}$, then $e_{0}$ is a trivialization of $-K_{\mathcal{F}_{\sigma}}$ outside $E \cup C$, where $E=p^{-1}([1: 0: 0])$ is the exceptional fiber and $C \cong C P^{1}$ is the line at infinity.

Let $W_{i}$ be open sets defined as follows:

$$
\begin{aligned}
& W_{0}=\left\{\left.\left(z_{1}, z_{2}\right)\left|4 \epsilon_{1}^{2}<\right| z_{1}\right|^{2}+\left|z_{2}\right|^{2}<\frac{1}{4 \epsilon_{1}^{2}}\right\}, \\
& W_{1}=\left\{\left.\left(z_{1}, z_{2}\right)\left|\epsilon_{1}^{2}<\right| z_{1}\right|^{2}+\left|z_{2}\right|^{2}<9 \epsilon_{1}^{2}\right\}, \\
& W_{2}=\left\{\left(a_{1}, a_{2}\right)|| a_{1}\left|<\epsilon_{2},\right| a_{2} \mid<1+\epsilon_{3}\right\}, \\
& W_{3}=\left\{\left(b_{1}, b_{2}\right)|| b_{1}\left|<\epsilon_{2},\right| b_{2} \mid<1+\epsilon_{3}\right\}, \\
& W_{4}=\left\{\left.\left(z_{1}, z_{2}\right)\left|\frac{1}{9 \epsilon_{1}^{2}}<\right| z_{1}\right|^{2}+\left|z_{2}\right|^{2}<\frac{1}{\epsilon_{1}^{2}}\right\}, \\
& W_{5}=\left\{\left(x_{1}, x_{2}\right)|| x_{1}\left|<\epsilon_{2},\right| x_{2} \mid<1+\epsilon_{3}\right\}, \\
& W_{6}=\left\{\left(u_{1}, u_{2}\right)|| u_{1}\left|<\epsilon_{2},\right| u_{2} \mid<1+\epsilon_{3}\right\},
\end{aligned}
$$

where $\epsilon_{1}$ and $\epsilon_{3}$ are small positive numbers. We may assume by appropriately choosing $\epsilon_{2}$ that $W_{1}, W_{2}$ and $W_{3}$ cover the set $p^{-1}\left(\left\{\left.\left(z_{1}, z_{2}\right)|| z_{1}\right|^{2}+\left|z_{2}\right|^{2}<9 \epsilon_{1}^{2}\right\}\right)$ and that $W_{4}, W_{5}$ and $W_{6}$ cover the set $\left\{\left.\left(z_{1}, z_{2}\right)|| z_{1}\right|^{2}+\left|z_{2}\right|^{2}>\frac{1}{9 \epsilon^{2}}\right\} \cup C$.

Let $e=\left\{e_{i}\right\}$ be the family of local trivializations given by setting

$$
\begin{array}{lll}
e_{0}=e_{1}=e_{4}=\frac{\partial}{\partial z_{1}} \wedge \frac{\partial}{\partial z_{2}}, & \\
e_{2}=\frac{\partial}{\partial a_{1}} \wedge \frac{\partial}{\partial a_{2}}, & e_{3}=\frac{\partial}{\partial b_{1}} \wedge \frac{\partial}{\partial b_{2}} \\
e_{5}=\frac{\partial}{\partial x_{1}} \wedge \frac{\partial}{\partial x_{2}}, & e_{6}=\frac{\partial}{\partial u_{1}} \wedge \frac{\partial}{\partial u_{2}} .
\end{array}
$$

These local trivializations are related by the formulae

$$
\begin{aligned}
e_{0} & =\frac{1}{a_{1}} \frac{\partial}{\partial a_{1}} \wedge \frac{\partial}{\partial a_{2}}=-\frac{1}{b_{1}} \frac{\partial}{\partial b_{1}} \wedge \frac{\partial}{\partial b_{2}} \\
& =x_{1}^{3} \frac{\partial}{\partial x_{1}} \wedge \frac{\partial}{\partial x_{2}}=-u_{1}^{3} \frac{\partial}{\partial u_{1}} \wedge \frac{\partial}{\partial u_{2}} .
\end{aligned}
$$

The connection form of a basic $Y$-connection with respect to $e=\left\{e_{i}\right\}$ is given as follows:

$$
\begin{array}{ll}
\widetilde{\zeta}_{0}=-\left(\lambda_{1}+\lambda_{2}\right) d t-\left(\delta_{1}+\delta_{2}\right) \omega, & \\
\widetilde{\zeta}_{1}=\widetilde{\zeta}_{4}=-\left(\lambda_{1}+\lambda_{2}\right) d t, & \widetilde{\zeta}_{3}=-\lambda_{1} d t, \\
\widetilde{\zeta}_{2}=-\lambda_{2} d t, & \widetilde{\zeta}_{6}=\left(2 \lambda_{1}-\lambda_{2}\right) d t \\
\widetilde{\zeta}_{5}=-\left(\lambda_{1}-2 \lambda_{2}\right) d t, &
\end{array}
$$


where

$$
\omega=\frac{1}{\left|z_{1}\right|^{2}+\left|z_{2}\right|^{2}}\left(\frac{1}{\delta_{1}} \bar{z}_{1} d z_{1}+\frac{1}{\delta_{2}} \bar{z}_{2} d z_{2}-\left(\frac{\lambda_{1}}{\delta_{1}}\left|z_{1}\right|^{2}+\frac{\lambda_{2}}{\delta_{2}}\left|z_{2}\right|^{2}\right) d t\right) .
$$

The family of functions $\widetilde{\alpha}$ is now as follows:

$$
\begin{array}{lll}
\widetilde{\alpha}_{12}=a_{1}^{-1}, & \widetilde{\alpha}_{13}=-b_{1}^{-1}, & \widetilde{\alpha}_{23}=-b_{2}, \\
\widetilde{\alpha}_{45}=x^{3}, & \widetilde{\alpha}_{46}=u^{3}, & \widetilde{\alpha}_{56}=-v^{3},
\end{array}
$$

and $\widetilde{\alpha}_{i j}=1$ for other indices. Consequently, the difference $\widetilde{\beta}$ of local connections is as follows:

$$
\begin{array}{ll}
\widetilde{\beta}_{01}=\widetilde{\beta}_{04}=\left(\delta_{1}+\delta_{2}\right) \omega, & \\
\widetilde{\beta}_{12}=\lambda_{1} d t-\frac{d a_{1}}{a_{1}}, & \widetilde{\beta}_{13}=\lambda_{2} d t-\frac{d b_{1}}{b_{1}}, \\
\widetilde{\beta}_{23}=-\left(\lambda_{1}-\lambda_{2}\right) d t+\frac{d b_{2}}{b_{2}}, & \\
\widetilde{\beta}_{45}=3 \lambda_{2} d t+3 \frac{d x}{x}, & \widetilde{\beta}_{46}=3 \lambda_{1} d t+3 \frac{d u}{u}, \\
\widetilde{\beta}_{56}=3\left(\lambda_{1}-\lambda_{2}\right) d t+3 \frac{d v}{v}, &
\end{array}
$$

and $\widetilde{\beta}_{i j}=0$ for other indices.

Precisely speaking, it is necessary to take refinements of the covering in order to assure $\log \alpha$, etc., are well-defined. However, in order to simplify the exposition, we keep our covering and leave the details to the reader.

The residue is now calculated as follows: first, we consider the neighborhood of the exceptional divisor $E$. The cocycle $B_{2}(\nabla, e)$ restricted to $\left\{W_{0}, W_{1}\right\}$ is equal to

$$
\begin{aligned}
& \zeta \cup(d \zeta+\beta)^{2} \\
= & c^{-3}\left(-\left(\lambda_{1}+\lambda_{2}\right) d t-\left(\delta_{1}+\delta_{2}\right) \omega\right) \cup\left(-\left(\delta_{1}+\delta_{2}\right) d \omega\right) \cup\left(\left(\delta_{1}+\delta_{2}\right) \omega\right) \\
= & c^{-3}\left(\lambda_{1}+\lambda_{2}\right)\left(\delta_{1}+\delta_{2}\right)^{2} d t \cup d \omega \cup \omega \\
= & -c^{-3}\left(\lambda_{1}+\lambda_{2}\right)\left(\delta_{1}+\delta_{2}\right)^{2} d t \wedge \omega \wedge d \omega,
\end{aligned}
$$

which is a 3 -form defined on $\left\{\left.\left(z_{1}, z_{2}\right)|| z_{1}\right|^{2}+\left|z_{2}\right|^{2}=4 \epsilon_{1}^{2}\right\}$, where $c=2 \pi \sqrt{-1}$. By taking the orientation into account, we see that its integration is equal to $\frac{\lambda_{1}+\lambda_{2}}{2 \pi \sqrt{-1}} \frac{\left(\delta_{1}+\delta_{2}\right)^{2}}{\delta_{1} \delta_{2}}$.

We now look at the cocycle $B_{2}(\nabla, e)$ restricted to $\left\{W_{1}, W_{2}, W_{3}\right\}$. Noticing that $d \zeta=0$ and that the only cochain involving $d t$ is $\zeta$, it suffices to consider the cochain

$$
\zeta \cup \beta^{2}+\Theta \cup \zeta \cup \beta+\Theta^{2} \cup \zeta \text {. }
$$

Before beginning the integration, remark that the orientation is determined as follows: first,

$$
\left(t, a_{1}, a_{2}\right) \sim\left(t, r_{1}, \theta_{1}, r_{2}, \theta_{2}\right) \sim\left(-r_{1}, t, \theta_{1}, r_{2}, \theta_{2}\right),
$$

where the sign $\sim$ means that the orientations are the same, and $\left(r_{i}, \theta_{i}\right)$ is the polar coordinate for the $a_{i}$-plane. Second, $\left(t, \theta_{1}, r_{2}, \theta_{2}\right) \sim\left(-r_{2},-t, \theta_{1}, \theta_{2}\right)$. Finally, $\left(-t, \theta_{1}, \theta_{2}\right) \sim\left(-\theta_{1},-t, \theta_{2}\right)$ and $\left(-t, \theta_{2}\right) \sim\left(-\theta_{2},-t\right)$. Hence $\left(-t, \theta_{1}, \theta_{2}\right),\left(-t, \theta_{2}\right)$ and 
$-t$ are adopted as the positive orientations on the pieces of honey-comb cells. Therefore, the integration is calculated as follows: first,

$$
\begin{aligned}
\int \zeta \cup \beta^{2} & =\int c^{-3}\left(-\left(\lambda_{1}+\lambda_{2}\right) d t\right) \cup\left(\lambda_{1} d t-\frac{d a_{1}}{a_{1}}\right) \cup\left(-\left(\lambda_{1}-\lambda_{2}\right) d t+\frac{d b_{2}}{b_{2}}\right) \\
& =\int c^{-3}\left(-\left(\lambda_{1}+\lambda_{2}\right) d t\right) \cup\left(-\frac{d a_{1}}{a_{1}}\right) \cup\left(-\frac{d a_{2}}{a_{2}}\right) \\
& =\int c^{-3}\left(\left(\lambda_{1}+\lambda_{2}\right) d t\right) \wedge\left(\frac{d a_{1}}{a_{1}}\right) \wedge\left(\frac{d a_{2}}{a_{2}}\right) \\
& =\frac{\lambda_{1}+\lambda_{2}}{2 \pi \sqrt{-1}} .
\end{aligned}
$$

Similarly,

$$
\begin{aligned}
\int \Theta \cup \zeta \cup \beta & =\int-c^{-2} \cdot(+1) \cdot\left(-\lambda_{2} d t\right) \wedge\left(-\frac{d a_{2}}{a_{2}}\right) \\
& =-c^{-1} \cdot(+1)\left(-\lambda_{2}\right) \\
& =\frac{\lambda_{2}}{2 \pi \sqrt{-1}}
\end{aligned}
$$

and

$$
\begin{aligned}
\int \Theta^{2} \cup \zeta & =\int c^{-1}(+1)(-1)\left(-\lambda_{1} d t\right) \\
& =\frac{\lambda_{1}}{2 \pi \sqrt{-1}}
\end{aligned}
$$

Hence the integration of the residue at $E$ along $S^{1}$ is equal to $\frac{2\left(\lambda_{1}+\lambda_{2}\right)}{2 \pi \sqrt{-1}}$.

The residue at $C$ is calculated in a similar way. By passing from $W_{0}$ to $W_{4}$, we obtain the value $-\frac{\lambda_{1}+\lambda_{2}}{2 \pi \sqrt{-1}} \frac{\left(\delta_{1}+\delta_{2}\right)^{2}}{\delta_{1} \delta_{2}}$. The rest of the integration is calculated as

$$
-9 \frac{\lambda_{1}+\lambda_{2}}{2 \pi \sqrt{-1}}-9 \frac{\lambda_{1}-2 \lambda_{2}}{2 \pi \sqrt{-1}}-9 \frac{-2 \lambda_{1}+\lambda_{2}}{2 \pi \sqrt{-1}}=0,
$$

where each term is calculated as in Example 7.2. Summing up, we obtained the following equations:

$$
\begin{aligned}
\operatorname{res} B_{2}\left(\mathcal{F}_{\sigma}, Y, e\right)_{E} & =\left(\frac{\lambda_{1}+\lambda_{2}}{2 \pi \sqrt{-1}} \frac{\left(\delta_{1}+\delta_{2}\right)^{2}}{\delta_{1} \delta_{2}}+\frac{2\left(\lambda_{1}+\lambda_{2}\right)}{2 \pi \sqrt{-1}}\right)[d t], \\
\operatorname{res} B_{2}\left(\mathcal{F}_{\sigma}, Y, e\right)_{C} & =-\frac{\lambda_{1}+\lambda_{2}}{2 \pi \sqrt{-1}} \frac{\left(\delta_{1}+\delta_{2}\right)^{2}}{\delta_{1} \delta_{2}}[d t] \\
B_{2}\left(\mathcal{F}_{\sigma}\right) & =\frac{2\left(\lambda_{1}+\lambda_{2}\right)}{2 \pi \sqrt{-1}}\left[\widetilde{C P^{2}}{ }_{\sigma}\right]
\end{aligned}
$$

where $[d t]$ and $\left[\widetilde{C P^{2}}{ }_{\sigma}\right]$ denote the fundamental cocycles.

EXAMPle 7.4. [15, Proposition 4.3] We continue with the setting of Example 7.3. Set $\lambda_{1}=\lambda_{2}=\frac{2 \pi \sqrt{-1}}{3}$, then the holonomy of $\mathcal{F}$ is given by the mapping 
$\left[z_{0}: z_{1}: z_{2}\right] \mapsto\left[z_{0}: e^{\frac{2 \pi \sqrt{-1}}{3}} z_{1}: e^{\frac{2 \pi \sqrt{-1}}{3}} z_{2}\right]$. We denote by $g$ this mapping. Let $q_{1}=[1: 1: 0], q_{2}=[1: 0: 1]$ and $q_{3}=[1: 1: 1]$ and consider the blow up $\widehat{M}$ of $\widetilde{C P^{2}}$ at the nine points $g^{i}\left(q_{1}\right), g^{i}\left(q_{2}\right), g^{i}\left(q_{3}\right), i=0,1,2$, then $g$ induces an automorphism $\hat{g}$ of $\widehat{M}$. It is not hard to see that the effect of blowing up at these points on $B_{2}\left(\mathcal{F}_{\widehat{g}}\right)$ is $\boldsymbol{Z}$-valued. Hence $F(\widehat{g})=\int_{M_{\widehat{g}}} B_{2}\left(\mathcal{F}_{\widehat{g}}\right)=\int_{M_{g}} B_{2}\left(\mathcal{F}_{g}\right)=\frac{4}{3}$. Note that $\widehat{M}$ is no longer Fano as is already mentioned in [15].

EXAMPLE 7.5. [24, section 3] Let $M$ be the manifold obtained by blowing up $\widetilde{\boldsymbol{C} P^{2}}$ at $[0: 1: 0]$. Let $g_{\lambda_{1}, \lambda_{2}}$ be the automorphism of $M$ induced by the diagonal matrix whose diagonal entries are $\left(1, e^{\lambda_{1}}, e^{\lambda_{2}}\right)$, viewed as an element of PGL(3; $\left.C\right)$. Then out of the exceptinal divisors, the situation is the same as in Example 7.3 and thus we may apply the same argument. Noticing that $[0: 1: 0]$ is the origin of the $u$-plane on which the vector field $T$ is equal to $-\lambda_{1} u_{1} \frac{\partial}{\partial u_{1}}+\left(\lambda_{2}-\lambda_{1}\right) u_{2} \frac{\partial}{\partial u_{2}}$, we see by repeating the same argument that the integration of the residue at the exceptional fiber over $[0: 1: 0]$ takes the value $2 \frac{-2 \lambda_{1}+\lambda_{2}}{2 \pi \sqrt{-1}}$. Hence, in total, $B_{2}\left(\mathcal{F}_{g_{\lambda_{1}, \lambda_{2}}}\right)=\frac{-2 \lambda_{1}+4 \lambda_{2}}{2 \pi \sqrt{-1}}\left[M_{g_{\lambda_{1}, \lambda_{2}}}\right]$. Now $F\left(g_{\lambda_{1}, \lambda_{2}}\right)=\int_{M_{g_{\lambda_{1}, \lambda_{2}}}} B_{2}\left(\mathcal{F}_{\lambda_{1}, \lambda_{2}}\right)$ is calculated as follows:

$$
\begin{array}{cc}
\left(\lambda_{1}, \lambda_{2}\right) & F\left(g_{\lambda_{1}, \lambda_{2}}\right) \\
(2 \pi \sqrt{-1} / p, 4 \pi \sqrt{-1} / p) & \frac{6}{p} \\
(2 \pi \sqrt{-1} / p, 0) & -\frac{2}{p} \\
(0,2 \pi \sqrt{-1} / p) & \frac{4}{p} \\
(-2 \pi \sqrt{-1} / p,-2 \pi \sqrt{-1} / p) & -\frac{2}{p}
\end{array}
$$

Note that the values $F\left(g_{\lambda_{1}, \lambda_{2}}\right)$ are additive with respect to $\left(\lambda_{1}, \lambda_{2}\right)$. It is also a consequence of the fact that the Futaki invariant is a group character.

The Futaki invariant can be defined even in the non-Kähler category. There is a vanishing result for a certain class of such manifolds.

TheOREM 7.6. The Futaki invariant of $S^{2 m-1} \times S^{2 n-1}$ with complex structures $\mathscr{C}$ constructed in [20] is trivial if $\mathscr{C}$ is linear.

Proof. The complex structure $\mathscr{C}$ on $S^{2 m-1} \times S^{2 n-1}$ is induced by a certain vector field $\xi$ which has a isolated singularity at the origin. If $\xi$ is of the form $\xi=$ $\sum_{i=1}^{m+n+1} \lambda_{i} z_{i} \frac{\partial}{\partial z_{i}}$, then the structure $\mathscr{C}$ is said to be linear (or diagonal). Under appropriate assumptions on $\lambda_{i}$, the standard embedding of $S^{2 m-1} \times S^{2 n-1}$ is transverse to $\xi$, and thus a complex structure is induced.

Let $\eta$ be the vector field given by $\eta=\sum_{i=1}^{m} \lambda_{i} z_{i} \frac{\partial}{\partial z_{i}}-\sum_{j=1}^{n} \lambda_{m+j} z_{m+j} \frac{\partial}{\partial z_{m+j}}$, then $\eta$ induces a non-vanishing holomorphic vector field on $S^{2 m-1} \times S^{2 n-1}$ because $[\xi, \eta]=0$ and $\xi, \eta$ are linearly independent on $\boldsymbol{C}^{m+n} \backslash\left(\boldsymbol{C}^{m} \times\{0\} \cup\{0\} \times \boldsymbol{C}^{n}\right)$. Noticing 
that the canonical bundle $K_{\left(S^{2 m-1} \times S^{2 n-1}, \mathscr{C}\right)}$ is differentiably trivial for $H^{2}\left(S^{2 m-1} \times\right.$ $\left.S^{2 n-1} ; Z\right)=0$, we see that $F$ is trivial by Corollary 6.7 .

REMARK 7.7. The complex structure $\mathscr{C}$ of $S^{2 m-1} \times S^{2 n-1}$ is known to be nonKähler for generic $\lambda_{i}[20]$.

Finally, we review some examples of complex codimension-one foliations found in [7] and [16] (cf. [2]).

EXAMPLE 7.8. Let $X_{\lambda}$ as in Example 7.3. Assume that $\lambda_{1} \lambda_{2} \neq 0$ and that $\lambda_{1} / \lambda_{2}$ is not a negative real number. Then it is known that $X_{\lambda}$ is transversal to $S^{3}$. Hence $X_{\lambda}$ induces a transversely holomorphic foliation $\mathcal{F}_{\lambda}$ of $S^{3}$, of complex codimension one. It is well-known that $B_{1}\left(\mathcal{F}_{\lambda}\right)=\frac{\left(\lambda_{1}+\lambda_{2}\right)^{2}}{\lambda_{1} \lambda_{2}}\left[S^{3}\right] \in H^{3}\left(S^{3} ; \boldsymbol{C} / \boldsymbol{Z}\right)$.

Let $C_{1}$ and $C_{2}$ be the closed leaves of $\mathcal{F}_{\lambda}$ passing through $(1,0)$ and $(0,1)$, respectively. Set $e=-\lambda_{2} \bar{z}_{2} \frac{\partial}{\partial z_{1}}+\lambda_{1} \bar{z}_{1} \frac{\partial}{\partial z_{2}}$, then $e$ is a trivialization of $-K_{\mathcal{F}_{\lambda}}$. On the other hand, let $Y$ be the vector field defined by $Y=\delta_{1} z_{1} \frac{\partial}{\partial z_{1}}+\delta_{2} z_{2} \frac{\partial}{\partial z_{2}}$, where $\lambda_{1} \delta_{2}-\lambda_{2} \delta_{1} \neq 0$, then $Y$ is a trivialization of $-K_{\mathcal{F}_{\lambda}}$ on $S^{3} \backslash\left\{C_{1} \cup C_{2}\right\}$. By using Example 7.1 , we see that $\pi_{1}$ res $B_{1}\left(\mathcal{F}_{\lambda}, X, e\right)=\left(\lambda_{2} / \lambda_{1}\right)\left[C_{1}\right]+\left(\lambda_{1} / \lambda_{2}\right)\left[C_{2}\right]$, where $\pi_{\text {! }}$ denotes the Thom isomorphism, i.e. $\pi_{!}: H_{c}^{3}\left(D_{i} ; C / Z\right) \stackrel{\sim}{\rightarrow} H^{1}\left(C_{i} ; C / Z\right)$, where $D_{i}$ is a small disc bundle over $C_{i}$. Thus $B_{1}\left(\mathcal{F}_{\lambda}\right)=\iota_{*}$ res $B_{1}\left(\mathcal{F}_{\lambda}, X, e\right)$. The imaginary part of this equation is shown in [2].

EXAMPLE 7.9. Examples 6.4(a) and 6.4(b) in [2] are also valid even if we take the real part into account. Let $X$ be a holomorphic vector field on $C^{2}$ of the form

$$
X=P\left(z_{1}, z_{2}\right) \frac{\partial}{\partial z_{1}}+Q\left(z_{1}, z_{2}\right) \frac{\partial}{\partial z_{2}}
$$

where $P$ and $Q$ are polynomials in $z_{1}$ and $z_{2}$ which have no common factors. Such a vector field defines a holomorphic foliation of $C P^{2}$ with isolated singularities which consist of a finite number of points, say, $\left\{p_{1}, \cdots, p_{n}\right\}$. We assume that these singularities are of Poincaré type, namely, the eigenvalues of the linear part $D X$ of $X$ at each singular point are independent over $\boldsymbol{R}$. Under these assumptions, we can choose for each $i$ a small open neighborhood $U_{i}$ of $p_{i}$ diffeomorphic to a ball so that the vector field $X$ induces non-singular transversely holomorphic foliations (in fact, flows) $\mathcal{F}_{i}$ of the spheres $S_{i}^{3}$ appearing as the boundary of $U_{i}$. We set $X_{n}=C P^{2} \backslash\left(\bigcup_{i=1}^{n} U_{i}\right)$, then by taking the double of $X_{n}$ we obtain a 4-dimensional manifold $M_{n}$ equipped with a transversely holomorphic foliation $\mathcal{F}$. We denote by $\iota_{i}$ the natural inclusion of $S_{i}^{3}$ to $M_{n}$, then $\mathcal{F}_{i}$ coincides with the pull-back of $\mathcal{F}$ by $\iota_{i}$. It is classically known that the Bott classes $\operatorname{Bott}_{1}\left(\mathcal{F}_{i}\right)$ of such foliations of $S_{i}^{3}$ are well-defined and that they are calculated as the residues of $c_{1}^{2}$ in the sense of Heitsch (more properly of Bott). These residues are written in terms of the Grothendieck residue, as was mentioned after Corollary 3.7 (see [4], also [18]). Indeed, Example 7.8 is a special case of this construction. We denote by res $c_{1}^{2}\left(p_{i}, X\right)$ the residue of $c_{1}^{2}$ at $p_{i}$. It is also shown in [4] that $\sum_{i=1}^{n} \operatorname{res}_{c_{1}^{2}}\left(p_{i}, X\right)=c_{1}^{2}\left(T C P^{2}-X\right)$, where $T C P^{2}-X$ is the virtual bundle. 
As $\operatorname{Bott}_{1}\left(\mathcal{F}_{i}\right)$ is well-defined, $B_{1}\left(\mathcal{F}_{i}\right)=\operatorname{res}_{c_{1}^{2}}\left(p_{i}, X\right) \bmod Z$ holds. Hence the sum $\sum_{i=1}^{n} B_{1}\left(\mathcal{F}_{i}\right)$ is equal to zero as an element of $\boldsymbol{C} / \boldsymbol{Z}$.

The rest of the argument is completely parallel. We can find by using the MeyerVietoris exact sequence a mapping $\tau=\oplus \tau_{i}: \bigoplus_{i=1}^{n} H^{3}\left(S_{i}^{3} ; \boldsymbol{C} / \boldsymbol{Z}\right) \stackrel{\tau}{\rightarrow} H^{4}\left(\boldsymbol{C} P^{2} ; \boldsymbol{C} / \boldsymbol{Z}\right)$ such that $H^{3}\left(M_{n} ; \boldsymbol{C} / \boldsymbol{Z}\right) \cong \operatorname{ker} \tau \cong(\boldsymbol{C} / \boldsymbol{Z})^{n-1}$ if $n>1$. Recalling that $\sum_{i=1}^{n} B_{1}\left(\mathcal{F}_{i}\right)=$ 0 in $\boldsymbol{C} / \boldsymbol{Z}$, the $n$-tuple $\left(B_{1}\left(\mathcal{F}_{1}\right), \cdots, B_{1}\left(\mathcal{F}_{n}\right)\right)$ naturally determines an element of $H^{3}\left(M_{n} ; \boldsymbol{C} / \boldsymbol{Z}\right)$ which is equal to $B_{1}(\mathcal{F})$. If $n=1$, then $H^{3}\left(M_{1} ; \boldsymbol{C} / \boldsymbol{Z}\right)=0$ and thus $B_{1}(\mathcal{F})$ must be trivial.

For example, consider again the vector field $X_{\lambda}$ on $C^{2}$ given by the formula

$$
X_{\lambda}=\lambda_{1} z_{1} \frac{\partial}{\partial z_{1}}+\lambda_{2} z_{2} \frac{\partial}{\partial z_{2}}
$$

and assume that $\lambda_{1} \lambda_{2} \neq 0$ and that $\lambda_{1} / \lambda_{2} \notin R$. Then the foliation of $C P^{2}$ induced from this vector field has three singular points $p_{1}:[0: 0: 1], p_{2}:[0: 1: 0]$ and $p_{3}:[1: 0: 0]$. Let $S_{1}^{3}, S_{2}^{3}$ and $S_{3}^{3}$ be the corresponding spheres. According to Example 7.8,

$$
\begin{aligned}
& B_{1}\left(\mathcal{F}_{1}\right)=\left(\frac{\lambda_{2}}{\lambda_{1}}+\frac{\lambda_{1}}{\lambda_{2}}\right) \operatorname{vol}_{S_{1}^{3}} \\
& B_{1}\left(\mathcal{F}_{2}\right)=\left(-\frac{\lambda_{2}}{\lambda_{1}-\lambda_{2}}-\frac{\lambda_{1}}{\lambda_{2}}\right) \operatorname{vol}_{S_{2}^{3}} \\
& B_{1}\left(\mathcal{F}_{3}\right)=\left(-\frac{\lambda_{1}}{\lambda_{2}-\lambda_{1}}-\frac{\lambda_{2}}{\lambda_{1}}\right) \operatorname{vol}_{S_{3}^{3}} .
\end{aligned}
$$

Note that $\tau_{1}\left(B_{1}\left(\mathcal{F}_{1}\right)\right)+\tau_{2}\left(B_{1}\left(\mathcal{F}_{2}\right)\right)+\tau\left(B_{1}\left(\mathcal{F}_{3}\right)\right)=\left(-\frac{\lambda_{2}}{\lambda_{1}-\lambda_{2}}\right)+\left(-\frac{\lambda_{1}}{\lambda_{2}-\lambda_{1}}\right)=1 \equiv 0$ in $\boldsymbol{C} / \boldsymbol{Z}$. These values clearly reflect the fact that the Julia component of $\mathcal{F}$ corresponding to $\frac{\lambda_{2}}{\lambda_{1}}$ of $B_{1}\left(\mathcal{F}_{1}\right)$ approaches to $p_{3}$ and yields again the same value to $B_{1}\left(\mathcal{F}_{3}\right)$. The same is true for other Julia components. The class $B_{1}(\mathcal{F})$ is given by the triple $\left(B_{1}\left(\mathcal{F}_{1}\right), B_{1}\left(\mathcal{F}_{2}\right), B_{1}\left(\mathcal{F}_{3}\right)\right)$ via the mapping $\tau$.

\section{REFERENCES}

[1] T. Asuke, A Remark on the Bott class, Ann. Fac. Sci. Toulouse, Vol. X, 1 (2001), pp. 5-21.

[2] - Residue and Localization of the Bott class, to appear in Topology.

[3] P. Baum And R. Bott, Singularities of Holomorphic Foliations, Jour. Diff. Geom., 7 (1972), pp. 279-342.

[4] R. BotT, A Residue Formula for Holomorphic Vector-fields, J. Diff. Geom., 1 (1967), pp. 311330.

[5] - On the Lefschetz formula and exotic characteristic classes, in Symposia Mathematica, Vol. X (Convegno di Geometria Differenziale, INDAM, Rome, 1971), Academic Press, London, 1972, pp. 95-105.

[6] R. Bott, S. Gilter, I. M. JAmes, Lectures on Algebraic and Differential Topology, Lecture Notes in Math. 279, Springer-Verlag, Berlin, 1972.

[7] R. Botт, L. W. Tu, Differential Forms in Algebraic Topology, Lecture Notes in Math., 82, Springer-Verlag, Berlin, 1995. 
[8] J. Cheeger, J. Simons, Differential characters and geometric invariants, in Geometry and topology (College Park, Md., 1983/84), Lecture Notes in Math., 1167, Springer-Verlag, Berlin, 1985, pp. 50-80.

[9] S. S. Chern, J. Simons, Chatacteristic forms and geometric invariants, Ann. of Math., 99, 1 (1974), pp. 48-69.

[10] G. Duminy, L'invariant de Godbillon-Vey d'un feuilletage se localise dans les feuilles ressort, unpublished preprint (1982).

[11] J. Dupont, Simplicial de Rham cohomology and Characteristic classes of flat bundles, Topology, 15 (1976), pp. 233-245.

[12] A. FUTAKI, An Obstruction to the Existence of Einstein Kähler Metrics, Invent. Math., 73, 3 (1983), pp. 437-443.

[13] A. FUTAKI, S. MORITA, Invariant polynomials of the automorphism group of a compact complex manifold, J. Differential Geom., 21, 1 (1985), pp. 135-142.

[14] A. FUTAKI, On a character of the automorphism group of a compact complex manifold, Invent. Math., 87, 3 (1987), pp. 655-660.

[15] A. FUTAKI, K. TSUBOI, Eta invariants and automorphisms of compact complex manifolds, in Recent topics in differential and analytic geometry, Adv. Stud. Pure Math., 18-I, Academic Press, Boston, MA, 1990, pp. 251-270.

[16] E. Ghys, X. Gomez-Mont, J. SAludes, Fatou and Julia Components of Transversely Holomorphic Foliations, in Essays on Geometry and Related Topics: Memoires dediés à André Haefliger, Monographie de l'Enseignement Mathématique 38 (edited by E. Ghys, P. de la Harpe, V. F. R. Jones, V. Sergiescu, T. Tsuboi), 2001, pp. 287-319.

[17] J. L. Heitsch, A Remark on the Integral Cohomology of $B \Gamma_{q}$, Topology, 11 (1972), pp. 141-146.

[18] — A Residue Formula for Holomorphic Foliations, Michigan Math. J., 27, 2 (1980), pp. 181-194.

[19] D. Lehmann, Variétés stratifiées $C^{\infty}$ : intégration de Čech-de Rham, et théorie de Chern-Weil, in Geometry and topology of submanifolds, II (Avignon, 1988), World Sci. Publishing, Teaneck, NJ, 1990, pp. 205-248.

[20] J. J. LoEв, M. Nicolau, Holomorphic flows and complex structures on products of odd dimensional spheres, Math. Ann., 306 (1996), pp. 781-817.

[21] E. Y. MilleR, de Rham cohomology with arbitrary coefficients, Topology, 17 (1978), pp. 193203.

[22] H. V. Pittie, Characteristic classes of foliations, Research Notes in Mathematics 10, Pitman Publishing, London-San Francisco, Calif.-Melbourne, 1976.

[23] T. SUWA, Indices of vector fields and residues of singular holomorphic foliations, Actualités Mathématiques, Hermann, Paris, 1998.

[24] K. TsuвoI, The lifted Futaki invariants and the Spin ${ }^{c}$-Dirac operators, Osaka J. Math., 32, 2 (1995), pp. 207-225. 